\title{
Sharp Conditions for Weighted 1-dimensional Poincaré Inequalities
}

\section{SENG-KEE CHUA \& RICHARD L. WHEEDEN}

ABSTRACT. We obtain necessary and sufficient conditions for all weighted one dimensional Poincaré inequalities. Weighted Hardy-type inequalities are an example of our estimates. We use these estimates to obtain some Poincaré type inequalities on product spaces for product weights. In some cases, we also obtain the exact constants in the inequalities. Finally, we apply our result to study solutions of ordinary differential equations with given average value.

1. Introduction. If $1 \leq q, p<\infty$, and $-\infty<a<b<\infty$, there is a constant $T_{p, q}$ depending on $a, b$ such that the one dimensional Poincaré type inequality

$$
\left(\int_{a}^{b}\left|f(x)-\frac{1}{b-a} \int_{a}^{b} f(t) d t\right|^{q} d x\right)^{1 / q} \leq T_{p, q}\left(\int_{a}^{b}\left|f^{\prime}(x)\right|^{p} d x\right)^{1 / p}
$$

holds for any Lipschitz continuous function $f$ on $[\mathrm{a}, \mathrm{b}]$. In this paper, we study the constant $T_{p, q}$ and, more generally, the problems of the validity of the corresponding weighted estimates

$$
\begin{gathered}
\left(\int_{a}^{b}\left|f(x)-\frac{1}{v[a, b]} \int_{a}^{b} f d v\right|^{q} \mu(x) d x\right)^{1 / q} \\
\leq C\left(\int_{a}^{b}\left|f^{\prime}(x)\right|^{p} w(x) d x\right)^{1 / p}
\end{gathered}
$$


and the value of the constant $C$. Here, $\mu, w$ are weights (i.e., nonnegative measurable functions which are finite a.e.) and $v$ is any nonnegative finite Borel measure on $[a, b]$. In fact, for any $1 \leq p, q<\infty$, we will give simple characterizations of $\mu, w, v$ in order for (1.2) to hold and estimates for the best constant. In some cases, we obtain the exact constant. We are primarily concerned with the case when $b-a<\infty$, but the results are also valid for unbounded intervals, if $f \in L_{v}^{1}[a, b]$ in addition to being Lipschitz continuous.

Estimates of type (1.2) are related to weighted Hardy inequalities of the form

$$
\left(\int_{a}^{b}\left|\int_{a}^{x} g(t) d t\right|^{q} \mu(x) d x\right)^{1 / q} \leq C\left(\int_{a}^{b}|g(x)|^{p} w(x) d x\right)^{1 / p}
$$

as well as the "dual" form

$$
\left(\int_{a}^{b}\left|\int_{x}^{b} g(t) d t\right|^{q} \mu(x) d x\right)^{1 / q} \leq C\left(\int_{a}^{b}|g(x)|^{p} w(x) d x\right)^{1 / p} .
$$

These Hardy estimates can be viewed as special cases of (1.2) applied to the indefinite integral of $g$, where $v$ is chosen to be the Delta measure at one of the endpoints $a$ or $b$. Weighted Hardy estimates have been studied extensively (see e.g., [25]), and the conditions which turn out to characterize the Poincaré estimate (1.2) have some similarity to those which characterize the Hardy estimates. For example, in the simple case when $1<p \leq q<\infty$ and all three measures are the same (i.e., when $\mu d x=w d x=d v$ ), the necessary and sufficient condition for (1.2) is

$$
\begin{aligned}
& \frac{1}{w[a, b]}\left(\sup _{a<x<b}\left\{w[x, b]^{1 / q}\left(\int_{a}^{x} w[a, t]^{p^{\prime}} w(t)^{1-p^{\prime}} d t\right)^{1 / p^{\prime}}\right\}\right. \\
& \left.+\sup _{a<x<b}\left\{w[a, x]^{1 / q}\left(\int_{x}^{b} w[t, b]^{p^{\prime}} w(t)^{1-p^{\prime}} d t\right)^{1 / p^{\prime}}\right\}\right)<\infty,
\end{aligned}
$$

whereas the known necessary and sufficient condition for (1.3) in the corresponding case is

$$
\sup _{a<x<b}\left\{w[x, b]^{1 / q}\left(\int_{a}^{x} w(t)^{1-p^{\prime}} d t\right)^{1 / p^{\prime}}\right\}<\infty,
$$

and the necessary and sufficient condition for the dual Hardy estimate (still with $1<p \leq q<\infty$ and $\mu=w)$ is

$$
\sup _{a<x<b}\left\{w[a, x]^{1 / q}\left(\int_{x}^{b} w(t)^{1-p^{\prime}} d t\right)^{1 / p^{\prime}}\right\}<\infty .
$$


The main difference is clearly the two factors $(w[a, t] / w[a, b])^{p^{\prime}}$, $(w[t, b] / w[a, b])^{p^{\prime}}$ in the integrands in the first condition above. As we shall see, the sufficiency of the first condition for the Poincaré estimate is fairly easy to deduce by using the corresponding Hardy estimates. On the other hand, the necessity is more complicated, and its proof occupies the majority of the paper.

Poincaré and Hardy inequalities, including their weighted generalized forms in higher dimensions, have had many important applications. There are many papers which study such inequalities, including results for other types of domains in higher dimensions and on manifolds: see for example [25], [5], [13], [21], [24], [30], and [22]. None of these give simple characterizing conditions for the measures in the Poincaré estimate (1.2) or for its analogues in higher dimensions, nor do they give good estimates for the best norm constants in (1.2). Some necessary and sufficient conditions for Poincaré inequalities have been given in terms of capacity (see [29], [36]), but those conditions are usually difficult to verify. Necessary and sufficient conditions have also been given in terms of measure of noncompactness (see [2], [17], [18], [19], and [20]). For weighted estimates, the questions of simple necessary and sufficient conditions and of best constants have been discussed mostly for Hardy inequalities, including estimates of the kind

$$
\|f\|_{L_{\mu}^{q}[a, b]} \leq C\left\|f^{\prime}\right\|_{L_{w}^{p}[a, b]},
$$

where $f$ is Lipschitz continuous and has compact support in $[a, b]$ (see, e.g., [25]). The smallest constant for an analogue of this estimate in higher dimensions with $\mu=w=1$ has been found in certain cases in [33], [34], [4], and [27]. However, it seems that for Poincaré type estimates (1.2), the smallest value of $T_{p, q}$ (i.e., the constant in the unweighted case (1.1)) had not been found until recently and then only in case $p=q=1$. In [28], a very elementary method is used to show that the best constant for $T_{1,1}$ is equal to $\frac{1}{2}(b-a)$.

Surprisingly, the method in [28] can be modified and generalized to obtain the best constant $T_{1, q}$ for $1<q<\infty$ in one dimension, and even the best constant for the weighted Poincaré inequality (1.2) when $p=1$. Moreover, it can be used together with the ideas in [25] to obtain reasonably good estimates for the best constant in all other cases of $p, q>1$, and it leads to simple necessary and sufficient conditions for (1.2).

Our results indicate that there are some basic differences between weighted Poincaré estimates in one dimension and in higher dimensions. For example, in the one dimensional case, our main result shows that the weight $w$ 
in (1.2) cannot vanish to arbitrarily high order at an interior point of $[a, b]$, in contrast to the situation in higher dimensions. See Remark 5 at the end of Section 1 for some further discussion of this point.

As corollaries of our one dimensional results, we obtain necessary and sufficient conditions for some special Poincaré estimates in higher dimensions; see Section 4. Also, in the Appendix, we use the one dimensional results to study solutions of some ordinary differential equations with given average value, as opposed to given initial value.

Acknowledgement. We would like to thank the referee for his helpful suggestions and for the correction of some mistakes.

In what follows, $C$ denotes various positive constants which may differ from place to place. Sometimes we will use $C(\alpha, \beta, \ldots)$ instead of $C$ to emphasize that a particular constant depends on $\alpha, \beta, \ldots$. By a weight $w$ we always mean a nonnegative measurable function that is finite almost everywhere. We will also denote the measure arising from $w$ by $w$, and sometimes we write $d w$ instead of $w(x) d x$. For any interval $[c, d]$, we set $\mu[c, d]=\int_{\mathcal{C}}^{d} \mu(x) d x$. We use the conventions that $1 / p+1 / p^{\prime}=1$ when $1 \leq p<\infty$, and that $0 \times \infty=0$.

The following theorem is the main result of the paper.

Theorem 1.4 Let $-\infty<a<b<\infty, \mu$ and $w$ be weights on $[a, b]$, and $v$ be a nonnegative finite Borel measure on $[a, b]$ with $v[a, b]>0$. Let $1 \leq p$, $q<\infty$, and define $K_{p, q}(\mu, v, w)$ in three ranges as follows:

$$
\begin{aligned}
& K_{1, q}(\mu, v, w)=\frac{1}{v[a, b]}\left\|\left(\mu[\cdot, b] v[a, \cdot]^{q}+\mu[a, \cdot] v[\cdot, b]^{q}\right)^{1 / q} w^{-1}\right\|_{L^{\infty}[a, b]}, \\
& K_{p, q}(\mu, v, w)=\frac{1}{v[a, b]}\left(\sup _{a<x<b}\left\{\mu[x, b]^{1 / q}\left(\int_{a}^{x} v[a, t]^{p^{\prime}} w(t)^{1-p^{\prime}} d t\right)^{1 / p^{\prime}}\right\}\right. \\
& \left.+\sup _{a<x<b}\left\{\mu[a, x]^{1 / q}\left(\int_{x}^{b} v[t, b]^{p^{\prime}} w(t)^{1-p^{\prime}} d t\right)^{1 / p^{\prime}}\right\}\right)
\end{aligned}
$$

if $1<p \leq q<\infty$, and if $1 \leq q<p<\infty$ and $1 / r=1 / q-1 / p$, let

$$
\begin{gathered}
K_{p, q}(\mu, v, w)=\frac{1}{v[a, b]}\left(\left\{\int_{a}^{b} \mu[x, b]^{r / a}\left(\int_{a}^{x} v[a, t]^{p^{\prime}} w(t)^{1-p^{\prime}} d t\right)^{r / q^{\prime}}\right.\right. \\
\left.\times v[a, x]^{p^{\prime}} w(x)^{1-p^{\prime}} d x\right\}^{1 / r}+
\end{gathered}
$$




$$
\begin{aligned}
& +\left\{\int_{a}^{b} \mu[a, x]^{r / a}\left(\int_{x}^{b} v[t, b]^{p^{\prime}} w(t)^{1-p^{\prime}} d t\right)^{r / q^{\prime}}\right. \\
& \left.\left.\times v[x, b]^{p^{\prime}} w(x)^{1-p^{\prime}} d x\right\}^{1 / r}\right) .
\end{aligned}
$$

Then

$$
\begin{gathered}
\left(\int_{a}^{b}\left|f(x)-\frac{1}{v[a, b]} \int_{a}^{b} f d v\right|^{q} \mu(x) d x\right)^{1 / q} \\
\leq C\left(\int_{a}^{b}\left|f^{\prime}(x)\right|^{p} w(x) d x\right)^{1 / p}
\end{gathered}
$$

for all Lipschitz continuous functions $f$ on $[a, b]$ if and only if $K_{p, q}(\mu, v, w)<$ $\infty$. Moreover, if $B_{p, q}(\mu, v, w)$ is the smallest possible constant $C$ in (1.5), then $K_{1, q}(\mu, v, w)=B_{1, q}(\mu, v, w)$ and

$$
\begin{aligned}
\frac{K_{p, q}(\mu, v, w)}{2} \leq B_{p, q}(\mu, v, w) \leq C_{0}(p, q) K_{p, q}(\mu, v, w) & \text { if } 1<p \leq q<\infty, \\
B_{p, q}(\mu, v, w) \leq C_{0}(p, q) K_{p, q}(\mu, v, w) & \text { if } 1 \leq q<p<\infty,
\end{aligned}
$$

where $C_{0}(p, q)=\left(1+q / p^{\prime}\right)^{1 / q}\left(1+p^{\prime} / q\right)^{1 / p^{\prime}}$ if $p \leq q$, and $C_{0}(p, q)=$ $q^{1 / q}\left(p^{\prime}\right)^{1 / q^{\prime}}$ if $q<p$.

Before proceeding, we make a few comments about this result. Some other comments are listed in the remarks at the end of this section. First, if $v$ is not a Delta measure, we will show (see case (ii) in the proof of the theorem) that if $K_{p, q}(\mu, v, w)<\infty$ for some $1 \leq p, q<\infty$, then $\mu \in L^{1}[a, b]$. Next, Theorem 1.4 has analogues in case $[a, b]$ is unbounded and also in case $p=1, q=\infty$; these are discussed in the remarks at the end of Section 2. Finally, although we have restricted ourselves to Lipschitz continuous functions, the result is still valid for absolutely continuous ones.

The definition of $K_{p, q}(\mu, v, w)$ in Theorem 1.4 is complicated because it depends on various ranges of $p$ and $q$, but it has a fairly simple form in each range. For easy comparison, we now state some useful propositions about Hardy-type inequalities obtained by combining some results in [25].

Proposition 1.6 Let $\mu$ and $w$ be weights on $[a, b],-\infty \leq a<b \leq \infty$. Let

$$
S(p, q, \mu, w)=\sup _{a<x<b} \mu[x, b]^{1 / q}\left\|w^{-1 / p}\right\|_{L^{p^{\prime}}[a, x]} \quad \text { if } 1 \leq p \leq q<\infty,
$$




$$
\begin{gathered}
S(p, q, \mu, w)=\left(\int_{a}^{b} \mu[x, b]^{r / q}\left(\int_{a}^{x} w(t)^{1-p^{\prime}} d t\right)^{r / q^{\prime}} w(x)^{1-p^{\prime}} d x\right)^{1 / r} \\
\text { if } 1 \leq q<p<\infty, 1 / r=1 / q-1 / p .
\end{gathered}
$$

Then

$$
\begin{aligned}
& \left(\int_{a}^{b}\left|\int_{a}^{x} f(t) d t\right|^{q} \mu(x) d x\right)^{1 / q} \\
& \quad \leq C\left(\int_{a}^{b}|f(x)|^{p} w(x) d x\right)^{1 / p} \quad \text { for all } f \in L_{w}^{p}[a, b]
\end{aligned}
$$

if and only if $S(p, q, \mu, w)<\infty$. Moreover, if $C_{B}$ is the best constant for the inequality, then $S(p, q, \mu, w) \leq C_{B} \leq C_{0}(p, q) S(p, q, \mu, w)$ if $p \leq q$ and $(q / r)^{1 / q^{\prime}} C_{0}(p, q) S(p, q, \mu, w) \leq C_{B} \leq C_{0}(p, q) S(p, q, \mu, w)$ if $q<p$, where $C_{0}(p, q)$ is as in Theorem 1.4 and $C_{0}(p, q)=1$ if either $p=1$ or $q=1$.

Furthermore, a similar theorem holds for $\int_{x}^{b} f(t) d t$ instead of $\int_{a}^{x} f(t) d t$ :

Proposition 1.7 Let $\mu$ and $w$ be weights on $[a, b],-\infty \leq a<b \leq \infty$. Let

$$
\begin{aligned}
& T(p, q, \mu, w)= \sup _{a<x<b} \mu[a, x]^{1 / q}\left\|w^{-1 / p}\right\|_{L^{p^{\prime}}[x, b]} \\
& \text { if } 1 \leq p \leq q<\infty, \text { and } \\
& T(p, q, \mu, w)=\left(\int_{a}^{b} \mu[a, x]^{r / q}\left(\int_{x}^{b} w(t)^{1-p^{\prime}} d t\right)^{r / q^{\prime}} w(x)^{1-p^{\prime}} d x\right)^{1 / r} \\
& \text { if } 1 \leq q<p<\infty, 1 / r=1 / q-1 / p .
\end{aligned}
$$

Then

$$
\begin{aligned}
& \left(\int_{a}^{b}\left|\int_{x}^{b} f(t) d t\right|^{q} \mu(x) d x\right)^{1 / q} \\
& \quad \leq C\left(\int_{a}^{b}|f(x)|^{p} w(x) d x\right)^{1 / p} \quad \text { for all } f \in L_{w}^{p}[a, b]
\end{aligned}
$$

if and only if $T(p, q, \mu, w)<\infty$. Moreover, if $C_{B}$ is the best constant for the inequality, then $T(p, q, \mu, w) \leq C_{B} \leq C_{0}(p, q) T(p, q, \mu, w)$ if $p \leq q$ and $(q / r)^{1 / q^{\prime}} C_{0}(p, q) T(p, q, \mu, w) \leq C_{B} \leq C_{0}(p, q) T(p, q, \mu, w)$ if $q<p$, where $C_{0}(p, q)$ is as in Theorem 1.4 and $C_{0}(p, q)=1$ if either $p=1$ or $q=1$. 
Even though it is assumed in [25] that the weights $\mu$ and $w$ in the previous two propositions are positive almost everywhere, it is not difficult to check that the propositions remain valid if $\mu$ and $w$ are just nonnegative.

The same proofs lead to the following analogues of these propositions obtained by replacing $f$ and $w$ by $f \tau$ and $w / \tau^{p}$ respectively.

Theorem 1.8 Let $\mu, \tau$, and $w$ be weights on $[a, b],-\infty \leq a<b \leq \infty$, and let

$$
\begin{aligned}
S(p, q, \mu, \tau, w)= & \sup _{a<x<b} \mu[x, b]^{1 / q}\left\|\tau w^{-1 / p}\right\|_{L^{p^{\prime}[a, x]}} \\
& \text { if } 1 \leq p \leq q<\infty, \text { and }
\end{aligned}
$$

$S(p, q, \mu, \tau, w)$

$$
\begin{gathered}
=\left(\int_{a}^{b} \mu[x, b]^{r / q}\left(\int_{a}^{x} \tau(t)^{p^{\prime}} w(t)^{1-p^{\prime}} d t\right)^{r / q^{\prime}} \tau(x)^{p^{\prime}} w(x)^{1-p^{\prime}} d x\right)^{1 / r} \\
\text { if } 1 \leq q<p<\infty, 1 / r=1 / q-1 / p .
\end{gathered}
$$

Then

$$
\begin{aligned}
& \left(\int_{a}^{b}\left|\int_{a}^{x} f(t) \tau(t) d t\right|^{q} \mu(x) d x\right)^{1 / q} \\
& \quad \leq C\left(\int_{a}^{b}|f(x)|^{p} w(x) d x\right)^{1 / p} \quad \text { for all } f \in L_{w}^{p}[a, b]
\end{aligned}
$$

if and only if $S(p, q, \mu, \tau, w)<\infty$. Moreover, if $C_{B}$ is the best constant for the inequality, then

$$
\begin{aligned}
S(p, q, \mu, \tau, w) & \leq C_{B} \leq C_{0}(p, q) S(p, q, \mu, \tau, w) \\
& \text { if } p \leq q, \\
(q / r)^{1 / q^{\prime}} C_{0}(p, q) S(p, q, \mu, \tau, w) \leq & C_{B} \leq C_{0}(p, q) S(p, q, \mu, \tau, w) \\
& \text { if } q<p,
\end{aligned}
$$

where $C_{0}(p, q)$ is as before.

Also, let

$$
\begin{aligned}
& T(p, q, \mu, \tau, w) \\
& \quad=\sup _{a<x<b} \mu[a, x]^{1 / q}\left\|\tau w^{-1 / p}\right\|_{L^{p^{\prime}}[x, b]} \quad \text { if } 1 \leq p \leq q<\infty,
\end{aligned}
$$


and

$$
\begin{aligned}
& T(p, q, \mu, \tau, w) \\
& =\left(\int_{a}^{b} \mu[a, x]^{r / q}\left(\int_{x}^{b} \tau(t)^{p^{\prime}} w(t)^{1-p^{\prime}} d t\right)^{r / q^{\prime}} \tau(x)^{p^{\prime}} w(x)^{1-p^{\prime}} d x\right)^{1 / r} \\
& \text { if } 1 \leq q<p<\infty, 1 / r=1 / q-1 / p
\end{aligned}
$$

Then

$$
\begin{aligned}
& \left(\int_{a}^{b}\left|\int_{x}^{b} f(t) \tau(t) d t\right|^{q} \mu(x) d x\right)^{1 / q} \\
& \quad \leq C\left(\int_{a}^{b}|f(x)|^{p} w(x) d x\right)^{1 / p} \quad \text { for all } f \in L_{w}^{p}[a, b]
\end{aligned}
$$

if and only if $T(p, q, \mu, \tau, w)<\infty$. Moreover, if $C_{B}$ is the best constant in the inequality, then

$$
\begin{array}{r}
T(p, q, \mu, \tau, w) \leq C_{B} \leq C_{0}(p, q) T(p, q, \mu, \tau, w) \\
\text { if } p \leq q,
\end{array}
$$

and

$$
\left(\frac{q}{r}\right)^{1 / q^{\prime}} C_{0}(p, q) T(p, q, \mu, \tau, w) \leq C_{B} \leq C_{0}(p, q) T(p, q, \mu, \tau, w)
$$

if $q<p$,

where $C_{0}(p, q)$ is as before.

Our proof of the sufficiency part of the main theorem is based on Theorem 1.8 together with the simple observation (see (2.1)) that

$$
\begin{aligned}
f(x)- & \frac{1}{v[a, b]} \int_{a}^{b} f d v \\
& =\frac{1}{v[a, b]}\left[\int_{a}^{x} v[a, z] f^{\prime}(z) d z-\int_{x}^{b} v[z, b] f^{\prime}(z) d z\right] .
\end{aligned}
$$

However, it turns out to be harder to prove the necessity part of Theorem 1.4 .

\section{Remarks 1.10.}

1. Our result allows the possibility that there exists a proper subinterval $[c, d]$ of $[a, b]$ such that $\mu(x)=0$ outside $[c, d]$. In that case, note that 


$$
\begin{aligned}
&\left(\int_{a}^{b}\left|f(x)-\frac{1}{v[a, b]} \int_{a}^{b} f d v\right|^{q} d \mu\right)^{1 / q} \\
&=\left(\int_{c}^{d}\left|f(x)-\frac{1}{v[a, b]} \int_{a}^{b} f d v\right|^{q} d \mu\right)^{1 / q},
\end{aligned}
$$

and that $1 /(v[a, b]) \int_{a}^{b} f d v$ may not be the average of $f$ over $[c, d]$.

2. Since

$$
\sup _{a<x<b} \mu[x, b]^{1 / a}\left\|v[a, .] w^{-1}\right\|_{L^{\infty}[a, x]}=\operatorname{esssup}_{a<x<b} \mu[x, b]^{1 / a} v[a, x] w(x)^{-1},
$$

and

$$
\sup _{a<x<b} \mu[a, x]^{1 / a}\left\|v[., b] w^{-1}\right\|_{L^{\infty}[x, b]}=\underset{a<x<b}{\operatorname{esssup}} \mu[a, x]^{1 / a} v[x, b] w(x)^{-1},
$$

the case $p=1$ of Theorem 1.4 is not very different from the case $p>1$, except that we are able to get the exact constant just as in [25].

3. It is possible to compute some values of $K_{1, q}(1,1,1)$ explicitly, and to estimate others. In fact, $K_{1, q}(1,1,1)=\frac{1}{2}(b-a)^{1 / q}$ for $1 \leq q \leq 3$ and $K_{1, q}(1,1,1)>\frac{1}{2}(b-a)^{1 / q}$ for $q>3$; see the appendix. Moreover, it is quite easy to check that $K_{1,4}(1,1,1)=(1 / 12)^{1 / 4}(b-a)^{1 / 4}$.

4. We can compare our conditions to some well-known weight conditions. First note that for any $1 \leq p, q<\infty$, we clearly have

$$
K_{p, q}(\mu, v, w) \leq 2 \mu[a, b]^{1 / q}\left\|w^{-1 / p}\right\|_{L^{p^{\prime}}[a, b]} .
$$

Hence it follows easily that $K_{p, p}(w, v, w) \leq C|b-a|$ if $w \in A_{p}$ (Muckenhoupt $A_{p}$ weights [13]) and $v$ is a finite nonnegative measure on $[a, b]$. Moreover, $K_{p, q}(\mu, v, w) \leq C|b-a|^{1 / q+1 / p^{\prime}}$ if $v$ is a finite nonnegative measure on $[a, b]$ and $(\mu, w) \in A_{p, q}$, i.e., if (see Torchinsky [35])

$$
\mu(I)^{1 / q}\left\|w^{-1 / p}\right\|_{L^{p^{\prime}(I)}} \leq C|I|^{1 / q+1 / p^{\prime}} \quad \text { for any interval } I \text { in } \mathbb{R} .
$$

However, even in the very special case when $\mu=w=v$ and $p=q$, our result provides us with many more weights than $A_{p}$ weights. In fact, we now know that (1.5) holds when $w=\mu=v$ and $p=q$ provided $w$ is in $L^{1}[a, b]$ and 


$$
\begin{aligned}
\sup _{a<x<b}\left\{w[x, b]^{1 / p}\left\|w[a, \cdot] w^{-1 / p}\right\|_{L^{p^{\prime}}[a, x]}\right. \\
\left.\quad+w[a, x]^{1 / p}\left\|w[\cdot, b] w^{-1 / p}\right\|_{L^{p^{\prime}}[x, b]}\right\}<\infty .
\end{aligned}
$$

Without the (bounded) factors $w[a, \cdot]$ and $w[\cdot, b]$, this would be valid for any $A_{p}$ weight $w$, but the presence of these factors allows other weights.

Similarly, let us show that when $1 \leq p \leq q<\infty$ and $w(t)=\mu(t)=$ $v(t)=|t-a|^{\alpha_{1}}|t-b|^{\alpha_{2}}$, then (1.5) holds if and only if $\alpha_{1}, \alpha_{2}>-1$ and $\alpha_{i}(1 / p-1 / q) \leq 1-(1 / p-1 / q)$ for $i=1,2$. It suffices to check the case $\mu(t)=v(t)=w(t)=|t-a|^{\alpha_{1}}$. First, if $p>1$, then by Theorem 1.4

$$
K_{p, q}(w, w, w)<\infty \quad \text { if and only if }
$$

both $\alpha_{1}>-1$ and $|x-a|^{\left(\alpha_{1}+1\right) / q}|x-a|^{\left[\alpha_{1}\left(1-p^{\prime}\right)+1\right] / p^{\prime}} \leq C<\infty$

for $x$ near $a$

(when $\alpha_{1}\left(1-p^{\prime}\right)+1=0$, we need $|x-a|^{\left(\alpha_{1}+1\right) / q}|\ln | x-a \mid \leq C$, which is always true for $x$ near $a$ since $\alpha_{1}>-1$ ), and this holds if and only if $\alpha_{1}>-1$ and $\left(\alpha_{1}+1\right)\left(1 / q+1 / p^{\prime}\right)-\alpha_{1} \geq 0$. Also, if $p=1$, then

$$
K_{1, q}(w, w, w)<\infty \quad \text { if and only if }
$$

both $\alpha_{1}>-1$ and $|x-a|^{\left(\alpha_{1}+1\right) / q} /|x-a|^{\alpha_{1}} \leq C<\infty$

for $x$ near $a$,

which holds if and only if $\left(\alpha_{1}+1\right) / q-\alpha_{1} \geq 0$. Hence, when $1 \leq p \leq$ $q<\infty$ and $w(t)=|t-a|^{\alpha_{1}}$, we obtain that

$$
K_{p, q}(w, w, w)<\infty \Longleftrightarrow \alpha_{1}>-1 \quad \text { and } \alpha_{1}\left(\frac{1}{p}-\frac{1}{q}\right) \leq 1-\left(\frac{1}{p}-\frac{1}{q}\right) \text {. }
$$

In particular, if $p=q$ and $w=\mu=v=|t-a|^{\alpha_{1}}|t-b|^{\alpha_{2}}$, the only restriction needed is $\alpha_{1}, \alpha_{2}>-1$.

5. From the observations in the previous remark, we see there are cases when the weight $w$ vanishes to high order at the endpoints $a, b$. However, it follows from the finiteness of $K_{p, q}(\mu, v, w)$ that if $\mu>0$ almost everywhere and $v[a, x], v[x, b]>0$ for all $a<x<b$, then $w^{1-p^{\prime}}$ is locally integrable in $(a, b)$, i.e., integrable away from the end points. In this case, $w$ cannot vanish at any interior point of $[a, b]$ to order 
$\geq p-1$ if $p>1$ (order $>0$ if $p=1$ ). This behavior is quite different from the situation in $\mathbb{R}^{n}$ when $n>1$, where $w$ can vanish to arbitrarily high order; see [10] and [22].

6. If $\mu[a, b]<\infty$, then

$$
\begin{aligned}
& \left(\int_{a}^{b}\left|f(x)-\frac{1}{\mu[a, b]} \int_{a}^{b} f(y) d \mu(y)\right|^{q} d \mu(x)\right)^{1 / q} \\
& \quad \leq 2\left(\int_{a}^{b}\left|f(x)-\frac{1}{v[a, b]} \int_{a}^{b} f d v\right|^{q} d \mu(x)\right)^{1 / q},
\end{aligned}
$$

and it follows that $K_{p, q}(\mu, \mu, w)<\infty$ if $K_{p, q}(\mu, v, w)<\infty$. Moreover, $K_{1, q}(\mu, \mu, w) \leq 2 K_{1, q}(\mu, v, w)$.

Our result implies that we have the following inequality:

Theorem 1.11 Let $1 \leq p, q<\infty$ and $\mu$ and $w$ be weights on $[a, b]$, and let $v$ be a nonnegative finite measure on $[a, b]$ with $v[a, b]>0$ such that $K_{p, q}(\mu, v, w)<\infty$. If $\mu[a, b]<\infty$, then

$$
\|f\|_{L_{\mu}^{a}[a, b]} \leq C\left\{\|f\|_{L_{v}^{1}[a, b]}+\left\|f^{\prime}\right\|_{L_{w}^{p}[a, b]}\right\}
$$

for all absolutely continuous functions $f$ on $[a, b]$.

2. Proof of the Main Theorem. Let $f$ be an absolutely continuous function on $[a, b]$. We have

$$
\begin{aligned}
& f(x)-\frac{1}{v[a, b]} \int_{a}^{b} f d v \\
& =\frac{1}{v[a, b]} \int_{a}^{b}[f(x)-f(y)] d v(y)=\frac{1}{v[a, b]} \int_{a}^{b} \int_{y}^{x} f^{\prime}(z) d z d v(y) \\
& =\frac{1}{v[a, b]}\left[\int_{a}^{x} \int_{y}^{x} f^{\prime}(z) d z d v(y)-\int_{x}^{b} \int_{x}^{y} f^{\prime}(z) d z d v(y)\right] \\
& =\frac{1}{v[a, b]}\left[\int_{a}^{x} v[a, z] f^{\prime}(z) d z-\int_{x}^{b} v[z, b] f^{\prime}(z) d z\right] \\
& =\frac{1}{v[a, b]}\left(\int_{a}^{b}\left\{v[a, z] f^{\prime}(z) x_{[a, x]}(z)-v[z, b] f^{\prime}(z) x_{[x, b]}(z)\right\} d z\right) .
\end{aligned}
$$

We now handle the case $p=1$. By Minkowski's integral inequality, 


$$
\begin{aligned}
\left(\int_{a}^{b} \mid f\right. & \left.(x)-\left.\frac{1}{v[a, b]} \int_{a}^{b} f d v\right|^{q} \mu(x) d x\right)^{1 / q} \\
& \leq \frac{1}{v[a, b]} \int_{a}^{b}\left[\int_{a}^{b}\left|v[a, z] f^{\prime}(z) x_{[a, x]}(z)-v[z, b] f^{\prime}(z) \chi_{[x, b]}(z)\right|^{q}\right. \\
& =\frac{1}{v[a, b]} \int_{a}^{b}\left[\int_{a}^{b}\left\{\left|v[a, z] f^{\prime}(z)\right|^{q} X_{[a, x]}(z)+\left|v[z, b] f^{\prime}(z)\right|^{q} X_{[x, b]}(z)\right\}\right. \\
& =\frac{1}{v[a, b]} \int_{a}^{b}\left[v[a, z]^{q} \mu[z, b]+v[z, b]^{q} \mu[a, z]\right]^{1 / q}\left|f^{\prime}(z)\right| d z \\
& =\frac{1}{v[a, b]} \int_{a}^{b} \frac{1}{w(z)}\left[v[a, z]^{q} \mu[z, b]+v[z, b]^{q} \mu[a, z]\right]^{1 / q} d z \\
& \leq K_{1, q}(\mu, v, w)\left(\int_{a}^{b}\left|f^{\prime}(z)\right| w(z) d z\right) .
\end{aligned}
$$

This proves (1.5) for $p=1$ with $C=K_{1, q}(\mu, v, w)$.

We now show that $K_{1, q}(\mu, v, w)$ is indeed the smallest constant. First, given weights $\mu, w$ and nonnegative measure $v$, suppose there exists $C_{B}>0$ such that

$$
\begin{gathered}
\left(\int_{a}^{b}\left|f(x)-\frac{1}{v[a, b]} \int_{a}^{b} f d v\right|^{q} \mu(x) d x\right)^{1 / q} \\
\quad \leq C_{B}\left(\int_{a}^{b}\left|f^{\prime}(x)\right| w(x) d x\right)
\end{gathered}
$$

for all absolutely continuous functions $f$ on $[a, b]$. For each $n \in \mathbf{N}$, let us define $w_{n}(x)=w(x)+1 / n$. Let $\alpha$ be a Lebesgue point of $1 / w_{n}$ such that $a<\alpha<b$ and $v[a, \alpha]+v[\alpha, b]=v[a, b]$ (the last equality holds except at most for a countable set of $\alpha$ ). Note that since at least one of $v[a, \alpha]$ or $v[\alpha, b]$ is strictly positive (recall that $v[a, b]>0$ by hypothesis), we may assume without loss of generality that $v[a, \alpha]>0$. Next, for any $0<\varepsilon<\min \{\alpha-a, b-\alpha\}$, let

$$
f_{\varepsilon}(x)= \begin{cases}-\int_{x}^{\alpha} \frac{v[\alpha, b]}{v[a, \alpha]} \frac{x_{[\alpha-\varepsilon, \alpha]}(t)}{\varepsilon w_{n}(t)} d t & \text { if } a \leq x \leq \alpha \\ \int_{\alpha}^{x} \frac{\chi_{[\alpha, \alpha+\varepsilon]}(t)}{\varepsilon w_{n}(t)} d t & \text { if } \alpha<x \leq b .\end{cases}
$$


Note that $v[a, \alpha-\varepsilon] \rightarrow v[a, \alpha)=v[a, \alpha], v[\alpha+\varepsilon, b] \rightarrow v(\alpha, b]=v[\alpha, b]$, and

$$
\frac{1}{\varepsilon} \int_{\alpha-\varepsilon}^{\alpha} w_{n}(t)^{-1} d t \quad \text { and } \quad \frac{1}{\varepsilon} \int_{\alpha}^{\alpha+\varepsilon} w_{n}(t)^{-1} d t \rightarrow w_{n}(\alpha)^{-1},
$$

since $\alpha$ is a Lebesgue point of $w_{n}^{-1}$. Hence as $\varepsilon \rightarrow 0$, we see that

$$
\begin{aligned}
& \frac{1}{v[a, b]} \int_{a}^{b} f_{\varepsilon} d v \rightarrow 0, \quad \text { and } \\
& \left(\int_{a}^{b}\left|f_{\varepsilon}(x)-\frac{1}{v[a, b]} \int_{a}^{b} f_{\varepsilon}(y) d v(y)\right|^{q} \mu(x) d x\right)^{1 / q} \\
& \quad \rightarrow\left(\left(\frac{v[\alpha, b]}{v[a, \alpha]}\right)^{q} \mu[a, \alpha]+\mu[\alpha, b]\right)^{1 / q} w_{n}(\alpha)^{-1}
\end{aligned}
$$

On the other hand,

$$
\begin{aligned}
\int_{a}^{b}\left|f_{\varepsilon}^{\prime}(z)\right| w_{n}(z) d z & =\frac{v[\alpha, b]}{v[a, \alpha]} \int_{\alpha-\varepsilon}^{\alpha} \frac{1}{\varepsilon} d x+\frac{1}{\varepsilon} \int_{\alpha}^{\alpha+\varepsilon} d x \\
& =\frac{v[\alpha, b]}{v[a, \alpha]}+1=\frac{v[a, b]}{v[a, \alpha]} .
\end{aligned}
$$

Since $w_{n} \geq w$, it is clear that (2.2) holds with $w_{n}$ instead of $w$. Hence

$$
C_{B} \geq \frac{\left(v[\alpha, b]^{q} \mu[a, \alpha]+\mu[\alpha, b] v[a, \alpha]^{q}\right)^{1 / q}}{w_{n}(\alpha) v[a, b]} .
$$

Thus $C_{B} \geq K_{1, q}\left(\mu, v, w_{n}\right)$. We now let $n \rightarrow \infty$ to obtain $C_{B} \geq K_{1, q}(\mu, v, w)$. This completes the proof when $p=1$.

We now consider the case $1<p<\infty$. First, by (2.1) we have

$$
\begin{aligned}
\left(\int_{a}^{b} \mid f(x)\right. & \left.-\left.\frac{1}{v[a, b]} \int_{a}^{b} f d v\right|^{q} \mu(x) d x\right)^{1 / q} \\
\leq & \frac{1}{v[a, b]}\left[\left(\int_{a}^{b}\left|\int_{a}^{x} v[a, z] f^{\prime}(z) d z\right|^{q} \mu(x) d x\right)^{1 / q}\right. \\
& \left.+\left(\int_{a}^{b}\left|\int_{x}^{b} v[z, b] f^{\prime}(z) d z\right|^{q} \mu(x) d x\right)^{1 / q}\right]
\end{aligned}
$$

$$
\begin{gathered}
\frac{C_{0}(p, q)}{v[a, b]}\{S(p, q, \mu, v[a, \cdot], w)+T(p, q, \mu, v[\cdot, b], w)\}\left\|f^{\prime}\right\|_{L_{w}^{p}[a, b]} \\
=C_{0}(p, q) K_{p, q}(\mu, v, w)\left\|f^{\prime}\right\|_{L_{w}^{p}[a, b]} \text { by Theorem } 1.8 .
\end{gathered}
$$


This proves (1.5) for $1<p<\infty, 1 \leq q<\infty$, with $C=C_{0}(p, q) K_{p, q}(\mu, v, w)$.

We will prove the converse by modifying the approach in [25]. Suppose $1<p<\infty, 1 \leq q<\infty$, and there exists $C_{B}>0$ such that

$$
\begin{gathered}
\left(\int_{a}^{b}\left|f(x)-\frac{1}{v[a, b]} \int_{a}^{b} f d v\right|^{q} \mu(x) d x\right)^{1 / q} \\
\leq C_{B}\left(\int_{a}^{b}\left|f^{\prime}(x)\right|^{p} w(x) d x\right)^{1 / p}
\end{gathered}
$$

for all Lipschitz continuous functions $f$ on $[a, b]$. Given any $n \in \mathbf{N}$, again let $w_{n}(x)=w(x)+1 / n$. For any $a<\alpha<b$, let

$$
\begin{aligned}
& f_{1}(x)=\int_{a}^{x} v[a, t]^{p^{\prime}-1} w_{n}(t)^{1-p^{\prime}} \chi_{[a, \alpha]}(t) d t, \\
& f_{2}(x)=\int_{a}^{x} v[t, b]^{p^{\prime}-1} w_{n}(t)^{1-p^{\prime}} \chi_{[\alpha, b]}(t) d t .
\end{aligned}
$$

Then, clearly, $f_{1}, f_{2}$ are Lipschitz continuous on $[a, b]$, and since $w_{n}>w$, we have (2.3) for $f_{1}$ and $f_{2}$, with $w$ replaced by $w_{n}$. Note that, by (2.1), the $q$-th power of the left-hand side of (2.3) with $f=f_{1}$ is

$$
\begin{aligned}
& \frac{1}{v[a, b]^{q}} \int_{a}^{b}\left|\int_{a}^{x} f_{1}^{\prime}(t) v[a, t] d t-\int_{x}^{b} f_{1}^{\prime}(t) v[t, b] d t\right|^{q} \mu(x) d x \\
& \geq \frac{1}{v[a, b]^{q}} \int_{\alpha}^{b}\left|\int_{a}^{x} f_{1}^{\prime}(t) v[a, t] d t\right|^{q} \mu(x) d x \text { as } f_{1}^{\prime}(t)=0 \text { for } t>\alpha \\
& \quad=\frac{1}{v[a, b]^{q}} \int_{\alpha}^{b}\left|\int_{a}^{\alpha} v[a, t]^{p^{\prime}} w_{n}(t)^{1-p^{\prime}} d t\right|^{q} \mu(x) d x \\
& \quad=\frac{1}{v[a, b]^{q}}\left(\int_{\alpha}^{b} \mu(x) d x\right)\left(\int_{a}^{\alpha} v[a, x]^{p^{\prime}} w_{n}(x)^{1-p^{\prime}} d x\right)^{q} .
\end{aligned}
$$

On the other hand, we have

$$
\int_{a}^{b}\left|f_{1}^{\prime}(x)\right|^{p} w_{n}(x) d x=\int_{a}^{\alpha} v[a, x]^{p^{\prime}} w_{n}(x)^{1-p^{\prime}} d x .
$$

Hence by (2.3) for $f_{1}$,

$$
v[a, b] C_{B} \geq\left(\int_{\alpha}^{b} \mu(x) d x\right)^{1 / q}\left(\int_{a}^{\alpha} v[a, x]^{p^{\prime}} w_{n}(x)^{1-p^{\prime}} d x\right)^{1 / p^{\prime}} .
$$

We now let $n \rightarrow \infty$ and see by the monotone convergence theorem that

(2.4) $\frac{1}{v[a, b]}\left(\int_{\alpha}^{b} \mu(x) d x\right)^{1 / q}\left(\int_{a}^{\alpha} v[a, x]^{p^{\prime}} w(x)^{1-p^{\prime}} d x\right)^{1 / p^{\prime}} \leq C_{B}$. 
By applying a similar argument to $f_{2}$, we obtain

(2.5) $\frac{1}{v[a, b]}\left(\int_{a}^{\alpha} \mu(x) d x\right)^{1 / q}\left(\int_{\alpha}^{b} v[x, b]^{p^{\prime}} w(x)^{1-p^{\prime}} d x\right)^{1 / p^{\prime}} \leq C_{B}$.

Hence, $C_{B} \geq \frac{1}{2} K_{p, q}(\mu, v, w)$ for the case $1<p \leq q<\infty$.

It remains only to show that (1.5) implies $K_{p, q}(\mu, v, w)<\infty$ when $1 \leq$ $q<p<\infty$. Suppose $1<q<p<\infty$ and recall that $1 / r=1 / q-1 / p$. Let us consider two cases:

Dase (i): $v=K \delta_{\alpha}$ with $a \leq \alpha \leq b, K>0$, i.e., $v$ is a Delta measure,

$$
v(E)= \begin{cases}K & \text { if } \alpha \in E, \\ 0 & \text { if } \alpha \notin E .\end{cases}
$$

In this case, note that by $(2.1)$,

$$
\begin{aligned}
& \int_{a}^{b}\left|f(x)-\int_{a}^{b} f d v / v[a, b]\right|^{q} \mu(x) d x \\
& \quad=\int_{a}^{\alpha}\left|\int_{x}^{\alpha} f^{\prime}(y) d y\right|^{q} \mu(x) d x+\int_{\alpha}^{b}\left|\int_{\alpha}^{x} f^{\prime}(y) d y\right|^{q} \mu(x) d x \\
& =\text { I + II. }
\end{aligned}
$$

Therefore,

$$
\mathrm{I}^{1 / q}, \mathrm{II}^{1 / q} \leq C_{B}\left(\int_{a}^{b}\left|f^{\prime}(y)\right|^{p} w(y) d y\right)^{1 / p}
$$

for all Lipschitz continuous functions on $[a, b]$. Hence,

$$
\begin{aligned}
\mathrm{I}^{1 / q} & \leq C_{B}\left(\int_{a}^{\alpha}\left|f^{\prime}(y)\right|^{p} w(y) d y\right)^{1 / p} \\
\mathrm{II}^{1 / q} & \leq C_{B}\left(\int_{\alpha}^{b}\left|f^{\prime}(y)\right|^{p} w(y) d y\right)^{1 / p}
\end{aligned}
$$

for all Lipschitz continuous functions on $[a, b]$. Thus, from the estimate for $I$,

(2.6) $\left(\int_{a}^{\alpha}\left|\int_{x}^{\alpha} g(y) d y\right|^{q} \mu(x) d x\right)^{1 / q} \leq C_{B}\left(\int_{a}^{\alpha}|g(y)|^{p} w(y) d y\right)^{1 / p}$ 
for all bounded functions $g$ on $[a, \alpha]$. Since any nonnegative function in $L_{w}^{p}[a, \alpha]$ is a pointwise limit of a monotone increasing sequence of bounded functions, (2.6) is then true for all functions $g$ in $L_{w}^{p}[a, \alpha]$. Hence by the Hardy-type inequalities (Propositions 1.6 and 1.7), we have

$\left(\frac{q}{r}\right)^{1 / q^{\prime}} C_{0}(p, q)\left(\int_{a}^{\alpha} \mu[a, x]^{r / q}\left[\int_{x}^{\alpha} w(t)^{1-p^{\prime}} d t\right]^{r / q^{\prime}} w(x)^{1-p^{\prime}} d x\right)^{1 / r} \leq C_{B}$.

Similarly, using the estimate for II, we have

$\left(\frac{q}{r}\right)^{1 / q^{\prime}} C_{0}(p, q)\left(\int_{\alpha}^{b} \mu[x, b]^{r / q}\left[\int_{\alpha}^{x} w(t)^{1-p^{\prime}} d t\right]^{r / q^{\prime}} w(x)^{1-p^{\prime}} d x\right)^{1 / r} \leq C_{B}$.

Since

$$
\frac{v[x, b]}{v[a, b]}=\left\{\begin{array}{ll}
1 & \text { if } a \leq x \leq \alpha \\
0 & \text { if } \alpha<x \leq b
\end{array} \text { and } \frac{v[a, x]}{v[a, b]}=\left\{\begin{array}{ll}
1 & \text { if } \alpha \leq x \leq b \\
0 & \text { if } a \leq x<\alpha
\end{array},\right.\right.
$$

we obtain

$$
\begin{gathered}
\left(\int_{a}^{\alpha} \mu[a, x]^{r / q}\left[\int_{x}^{\alpha} w(t)^{1-p^{\prime}} d t\right]^{r / q^{\prime}} w(x)^{1-p^{\prime}} d x\right)^{1 / r} \\
+\left(\int_{\alpha}^{b} \mu[x, b]^{r / q}\left[\int_{\alpha}^{x} w(t)^{1-p^{\prime}} d t\right]^{r / q^{\prime}} w(x)^{1-p^{\prime}} d x\right)^{1 / r} \\
=K_{p, q}(\mu, v, w) .
\end{gathered}
$$

Hence $K_{p, q}(\mu, v, w) \leq C C_{B}<\infty$.

- Case (ii): $v$ is not a Delta measure. Then (recall that $v$ is nonnegative) clearly there exists $a \leq \alpha_{0}<b$ such that $0<v\left[a, \alpha_{0}\right]<v[a, b]$. Hence by choosing an appropriate $\alpha_{0}<\alpha<b$, we have $v[a, \alpha)$ and $v(\alpha, b]>0$. Thus

$$
\int_{\alpha}^{b} v[x, b]^{p^{\prime}} w(x)^{1-p^{\prime}} d x, \quad \int_{a}^{\alpha} v[a, x]^{p^{\prime}} w(x)^{1-p^{\prime}} d x>0 .
$$

Hence by (2.4) and (2.5), we have $\int_{a}^{b} \mu(x) d x<\infty$.

Let us now show that the first part of $K_{p, q}(\mu, v, w)$ is finite, i.e., that

$$
\begin{gathered}
\int_{a}^{b} \mu[x, b]^{r / a}\left(\int_{a}^{x} v[a, t]^{p^{\prime}} w(t)^{1-p^{\prime}} d t\right)^{r / q^{\prime}} \\
v[a, x]^{p^{\prime}} w(x)^{1-p^{\prime}} d x<\infty .
\end{gathered}
$$


We may assume that $\mu[a, b]>0$. Let $a<b_{0} \leq b$ such that $\mu\left[b_{0}, b\right]=0$ and $\mu[x, b]>0$ for $a \leq x<b_{0}$. If

$$
\int_{a}^{b_{0}} v[a, x]^{p^{\prime}} w(x)^{1-p^{\prime}} d x=0,
$$

then the left-hand side of (2.7) is equal to zero. Now, suppose

$$
\int_{a}^{b_{0}} v[a, x]^{p^{\prime}} w(x)^{1-p^{\prime}} d x>0 .
$$

Then there exists $a<\alpha<b_{0}$ such that

$$
\int_{a}^{\alpha} v[a, x]^{p^{\prime}} w(x)^{1-p^{\prime}} d x>0 .
$$

We will prove (2.7) by showing that both

$$
\begin{aligned}
& \int_{\alpha}^{b} \mu[x, b]^{r / q}\left(\int_{a}^{x} v[a, t]^{p^{\prime}} w(t)^{1-p^{\prime}} d t\right)^{r / q^{\prime}} v[a, x]^{p^{\prime}} w(x)^{1-p^{\prime}} d x<\infty, \\
& \int_{a}^{\alpha} \mu[x, b]^{r / q}\left(\int_{a}^{x} v[a, t]^{p^{\prime}} w(t)^{1-p^{\prime}} d t\right)^{r / q^{\prime}} v[a, x]^{p^{\prime}} w(x)^{1-p^{\prime}} d x<\infty .
\end{aligned}
$$

By (2.8), there exists $a<a^{\prime}<\alpha$ such that $v\left[a, a^{\prime}\right]>0$. For each $n \in \mathbf{N}$, we let $b_{n}=b_{0}-\left(b_{0}-\alpha\right) /(2 n)$. First note that $b_{n}>\alpha$, and

$$
\begin{gathered}
\int_{a}^{b_{n}} \mu[x, b]^{r / q}\left(\int_{a}^{x} v[a, t]^{p^{\prime}} w(t)^{1-p^{\prime}} d t\right)^{r / q^{\prime}} v[a, x]^{p^{\prime}} w(x)^{1-p^{\prime}} d x \\
\leq \mu[a, b]^{r / q} \int_{a}^{b_{n}}\left(\int_{a}^{x} v[a, t]^{p^{\prime}} w(t)^{1-p^{\prime}} d t\right)^{r / q^{\prime}} \\
\quad \leq C \mu[a, b]^{r / q}\left(\int_{a}^{b_{n}} v[a, t]^{p^{\prime}} w(t)^{1-p^{\prime}} d t\right)^{1+\left(r / q^{\prime}\right)}<\infty
\end{gathered}
$$

because $\mu[a, b]<\infty$ and the second factor in (2.11) is finite by (2.4) applied with $\alpha=b_{n}$ there, since the fact that $b_{n}<b_{0}$ implies $\mu\left[b_{n}, b\right]>0$. Thus, it is now clear that (2.10) holds.

We now show that (2.9) holds. Note that this will follow if we show that

$$
\begin{array}{r}
\int_{\alpha}^{b} \mu[x, b]^{r / q}\left(\int_{\alpha}^{x} v[a, t]^{p^{\prime}} w(t)^{1-p^{\prime}} d t\right)^{r / q^{\prime}} v[a, x]^{p^{\prime}} w(x)^{1-p^{\prime}} d x<\infty, \\
\int_{\alpha}^{b} \mu[x, b]^{r / q} v[a, x]^{p^{\prime}} w(x)^{1-p^{\prime}} d x<\infty,
\end{array}
$$


since

$$
\int_{a}^{\alpha} v[a, t]^{p^{\prime}} w(t)^{1-p^{\prime}} d t<\infty
$$

Now recall that $w_{n}(x)=w(x)+1 / n$ and let

$$
\begin{aligned}
& g_{n}^{\prime}(x)= \\
& =\mu[x, b]^{r / p q}\left(\int_{\alpha}^{x} v[a, t]^{p^{\prime}} w_{n}(t)^{1-p^{\prime}} d t\right)^{r / p q^{\prime}} v[a, x]^{p^{\prime}-1} w_{n}(x)^{1-p^{\prime}} \chi_{\left[\alpha, b_{n}\right]}(x) \\
& +K_{n} v[a, x]^{p^{\prime}-1} w_{n}(x)^{1-p^{\prime}} \chi_{\left[a^{\prime}, \alpha\right]}(x), \\
& h_{n}^{\prime}(x)= \\
& =\mu[x, b]^{r / p q} v[a, x]^{p^{\prime}-1} w_{n}(x)^{1-p^{\prime}} \chi_{\left[\alpha, b_{n}\right]}(x) \\
& +J_{n} v[a, x]^{p^{\prime}-1} w_{n}(x)^{1-p^{\prime}} \chi_{\left[a^{\prime}, \alpha\right]}(x),
\end{aligned}
$$

where $K_{n}, J_{n}$ are finite constants chosen so that (clearly, $g_{n}$ and $h_{n}$ are Lipschitz continuous on $[a, b]$ )

$$
\begin{aligned}
& \int_{a}^{\alpha} g_{n}^{\prime}(x) v[a, x] d x=2 \int_{\alpha}^{b} g_{n}^{\prime}(x) v[x, b] d x \\
& \int_{a}^{\alpha} h_{n}^{\prime}(x) v[a, x] d x=2 \int_{\alpha}^{b} h_{n}^{\prime}(x) v[x, b] d x
\end{aligned}
$$

(note that $\int_{a^{\prime}}^{\alpha} v[a, x]^{p^{\prime}} w_{n}(x)^{1-p^{\prime}} d x>0$ as $v\left[a, a^{\prime}\right]>0$ ). Then

$$
\begin{aligned}
& \frac{K_{n}=}{2 \int_{\alpha}^{b_{n}} \mu[x, b]^{r / p q}\left(\int_{\alpha}^{x} v[a, t]^{p^{\prime}} w_{n}(t)^{1-p^{\prime}} d t\right)^{r / p q^{\prime}}} \\
\int_{a^{\prime}}^{\alpha} v[a, x]^{p^{\prime}} w_{n}(x)^{1-p^{\prime}} d x & \frac{v[a, x]^{p^{\prime}-1} w_{n}(x)^{1-p^{\prime}} v[x, b] d x}{\int_{a^{\prime}}^{\alpha} v[a, x]^{p^{\prime}} w_{n}(x)^{1-p^{\prime}} d x}
\end{aligned}
$$




$$
\begin{gathered}
\leq \frac{2\left\{\int_{\alpha}^{b_{n}} \mu[x, b]^{r / q}\left(\int_{\alpha}^{x} v[a, t]^{p^{\prime}} w_{n}(t)^{1-p^{\prime}} d t\right)^{r / q^{\prime}} v[a, x]^{p^{\prime}} w_{n}(x)^{1-p^{\prime}} d x\right\}^{1 / p}}{\int_{a^{\prime}}^{\alpha} v[a, x]^{p^{\prime}} w_{n}(x)^{1-p^{\prime}} d x} \\
\leq \frac{2\left\{\int_{\alpha}^{b_{n}} v[x, b]^{p^{\prime}} w_{n}(x)^{1-p^{\prime}} d x\right)^{1 / p^{\prime}} \text { by Hölder's inequality }}{\left.\leq 2[x, b]^{r / q}\left(\int_{\alpha}^{x} v[a, t]^{p^{\prime}} w_{n}(t)^{1-p^{\prime}} d t\right)^{r / q^{\prime}} v[a, x]^{p^{\prime}} w_{n}(x)^{1-p^{\prime}} d x\right\}^{1 / p}} \\
\int_{a^{\prime}}^{\alpha} v[a, x]^{p^{\prime}} w_{n}(x)^{1-p^{\prime}} d x \\
\cdot\left\{\int_{\alpha}^{b} v[x, b]^{p^{\prime}} w(x)^{1-p^{\prime}} d x\right\}^{1 / p^{\prime}}
\end{gathered}
$$

and

$$
J_{n} \leq \frac{2\left\{\int_{\alpha}^{b_{n}} \mu[x, b]^{r / q} v[a, x]^{p^{\prime}} w_{n}(x)^{1-p^{\prime}} d x\right\}^{1 / p}\left\{\int_{\alpha}^{b} v[x, b]^{p^{\prime}} w(x)^{1-p^{\prime}} d x\right\}^{1 / p^{\prime}}}{\int_{a^{\prime}}^{\alpha} v[a, x]^{p^{\prime}} w_{n}(x)^{1-p^{\prime}} d x} .
$$

We now show that $\int_{\alpha}^{b} v[x, b]^{p^{\prime}} w(x)^{1-p^{\prime}} d x<\infty$. To this end, first observe that there exists $a<\beta<b$ such that $\mu[a, \beta], \mu[\beta, b]>0$. Now if $\beta>\alpha$, we have

$$
\begin{aligned}
\int_{\alpha}^{b} v[x, b]^{p^{\prime}} w(x)^{1-p^{\prime}} d x= & \int_{\alpha}^{\beta} v[x, b]^{p^{\prime}} w(x)^{1-p^{\prime}} d x \\
& +\int_{\beta}^{b} v[x, b]^{p^{\prime}} w(x)^{1-p^{\prime}} d x
\end{aligned}
$$

and

$$
\begin{aligned}
\int_{\alpha}^{\beta} v[x, b]^{p^{\prime}} w(x)^{1-p^{\prime}} d x & \leq v[a, b]^{p^{\prime}} \int_{\alpha}^{\beta} w(x)^{1-p^{\prime}} d x \\
& \leq(v[a, b] / v[a, \alpha])^{p^{\prime}} \int_{\alpha}^{\beta} v[a, x]^{p^{\prime}} w(x)^{1-p^{\prime}} d x \\
& \leq C \int_{a}^{\beta} v[a, x]^{p^{\prime}} w(x)^{1-p^{\prime}} d x<\infty,
\end{aligned}
$$

by (2.4) since $\mu[\beta, b]>0$. Moreover, $\int_{\beta}^{b} v[x, b]^{p^{\prime}} w(x)^{1-p^{\prime}} d x<\infty$ by $(2.5)$ and the fact that $\mu[a, \beta]>0$. Finally, if $\alpha \geq \beta$, then

$$
\int_{\alpha}^{b} v[x, b]^{p^{\prime}} w(x)^{1-p^{\prime}} d x \leq \int_{\beta}^{b} v[x, b]^{p^{\prime}} w(x)^{1-p^{\prime}} d x<\infty
$$


as we just observed.

Next note that

$$
\text { (2.13) } \begin{aligned}
& \int_{a}^{b}\left|g_{n}^{\prime}(x)\right|^{p} w_{n}(x) d x \\
= & \int_{\alpha}^{b}\left|g_{n}^{\prime}(x)\right|^{p} w_{n}(x) d x+\int_{a}^{\alpha}\left|g_{n}^{\prime}(x)\right|^{p} w_{n}(x) d x \\
= & \int_{\alpha}^{b_{n}} \mu[x, b]^{r / a}\left(\int_{\alpha}^{x} v[a, t]^{p^{\prime}} w_{n}(t)^{1-p^{\prime}} d t\right)^{r / q^{\prime}} v[a, x]^{p^{\prime}} w_{n}(x)^{1-p^{\prime}} d x \\
& \quad+K_{n}^{p} \int_{a^{\prime}}^{\alpha} v[a, x]^{p^{\prime}} w_{n}(x)^{1-p^{\prime}} d x \\
\leq & \left\{\int_{\alpha}^{b_{n}} \mu[x, b]^{r / a}\left(\int_{\alpha}^{x} v[a, t]^{p^{\prime}} w_{n}(t)^{1-p^{\prime}} d t\right)^{r / q^{\prime}} v[a, x]^{p^{\prime}} w_{n}(x)^{1-p^{\prime}} d x\right\} \\
& \cdot\left(1+2^{p} T\right)
\end{aligned}
$$

by our estimate for $K_{n}$, where (since $\left.w_{n} \leq w_{1}\right)$

$$
T=\left(\frac{\int_{\alpha}^{b} v[x, b]^{p^{\prime}} w(x)^{1-p^{\prime}} d x}{\int_{a^{\prime}}^{\alpha} v[a, x]^{p^{\prime}} w_{1}(x)^{1-p^{\prime}} d x}\right)^{p / p^{\prime}}<\infty .
$$

Similarly, we can show that

$$
\int_{a}^{b}\left|h_{n}^{\prime}(x)\right|^{p} w_{n}(x) d x \leq\left\{\int_{\alpha}^{b_{n}} \mu[x, b]^{r / q} v[a, x]^{p^{\prime}} w_{n}(x)^{1-p^{\prime}} d x\right\}\left(1+2^{p} T\right) .
$$

On the other hand, note that $g_{n}^{\prime} \geq 0$ and

$$
\begin{aligned}
& \int_{\alpha}^{b}\left|\int_{a}^{x} g_{n}^{\prime}(t) v[a, t] d t\right|^{q} \mu(x) d x \\
= & q \int_{\alpha}^{b} \int_{a}^{x}\left[\int_{a}^{y} g_{n}^{\prime}(t) v[a, t] d t\right]^{q-1} g_{n}^{\prime}(y) v[a, y] d y \mu(x) d x \\
= & q \int_{a}^{b}\left(\int_{\max \{y, \alpha\}}^{b} \mu\right)\left(\int_{a}^{y} g_{n}^{\prime}(t) v[a, t] d t\right)^{q-1} g_{n}^{\prime}(y) v[a, y] d y \\
\geq & q \int_{\alpha}^{b} \mu[y, b]\left(\int_{a}^{y} g_{n}^{\prime}(t) v[a, t] d t\right)^{q-1} g_{n}^{\prime}(y) v[a, y] d y .
\end{aligned}
$$

Moreover, if $\alpha \leq y \leq b_{n}$, 
(2.15) $\int_{a}^{y} g_{n}^{\prime}(t) v[a, t] d t$

$$
\begin{gathered}
\geq \int_{\alpha}^{y} \mu[t, b]^{r /(p q)}\left(\int_{\alpha}^{t} v[a, z]^{p^{\prime}} w_{n}(z)^{1-p^{\prime}} d z\right)^{r /\left(p q^{\prime}\right)} \\
\times v[a, t]^{p^{\prime}} w_{n}(t)^{1-p^{\prime}} d t \\
\geq \mu[y, b]^{r /(p q)} \int_{\alpha}^{y}\left(\int_{\alpha}^{t} v[a, z]^{p^{\prime}} w_{n}(z)^{1-p^{\prime}} d z\right)^{r /\left(p q^{\prime}\right)} \\
\times v[a, t]^{p^{\prime}} w_{n}(t)^{1-p^{\prime}} d t \\
=\left(1+\frac{r}{p q^{\prime}}\right)^{-1} \mu[y, b]^{r /(p q)}\left(\int_{\alpha}^{y} v[a, t]^{p^{\prime}} w_{n}(t)^{1-p^{\prime}} d t\right)^{r /\left(p q^{\prime}\right)+1} .
\end{gathered}
$$

By (2.1),

$$
\begin{aligned}
v & {[a, b]^{q} \int_{a}^{b}\left|g_{n}(x)-\frac{1}{v[a, b]} \int_{a}^{b} g_{n} d v\right|^{q} \mu(x) d x } \\
& =\int_{a}^{b}\left|\int_{a}^{x} g_{n}^{\prime}(t) v[a, t] d t-\int_{x}^{b} g_{n}^{\prime}(t) v[t, b] d t\right|^{q} \mu(x) d x \\
& \geq \int_{\alpha}^{b}\left|\int_{a}^{x} g_{n}^{\prime}(t) v[a, t] d t-\int_{x}^{b} g_{n}^{\prime}(t) v[t, b] d t\right|^{q} \mu(x) d x \\
& \geq \frac{1}{2^{q}} \int_{\alpha}^{b}\left|\int_{a}^{x} g_{n}^{\prime}(t) v[a, t] d t\right|^{q} \mu(x) d x \quad \text { by }(2.12) \\
& \geq C \int_{\alpha}^{b_{n}} \mu[y, b]^{r / q}\left(\int_{\alpha}^{y} v[a, t]^{p^{\prime}} w_{n}(t)^{1-p^{\prime}} d t\right)^{r / q^{\prime}} \\
\times & \times v[a, y]^{p^{\prime}} w_{n}(y)^{1-p^{\prime}} d y,
\end{aligned}
$$

by (2.14), (2.15), the definition of $g_{n}^{\prime}$ and the fact that

$$
\frac{1}{r}=\frac{1}{q}-\frac{1}{p}
$$

Now, (2.3) applied to the function $g_{n}$, together with (2.13) and (2.16), implies that 
(2.17) $v[a, b] C_{B}$

$$
\begin{aligned}
& \geq C \frac{\left\{\int_{\alpha}^{b_{n}} \mu[x, b]^{r / q}\left(\int_{\alpha}^{x} v[a, t]^{p^{\prime}} w_{n}(t)^{1-p^{\prime}} d t\right)^{r / q^{\prime}} v[a, x]^{p^{\prime}} w_{n}(x)^{1-p^{\prime}} d x\right\}^{1 / q}}{\left\{\int_{\alpha}^{b_{n}} \mu[x, b]^{r / q}\left(\int_{\alpha}^{x} v[a, t]^{p^{\prime}} w_{n}(t)^{1-p^{\prime}} d t\right)^{r / q^{\prime}} v[a, x]^{p^{\prime}} w_{n}(x)^{1-p^{\prime}} d x\right\}^{1 / p}} \cdot \frac{1}{\left(1+2^{p} T\right)^{1 / p}} \\
& =C\left\{\int_{\alpha}^{b_{n}} \mu[x, b]^{r / q}\left(\int_{\alpha}^{x} v[a, t]^{p^{\prime}} w_{n}(t)^{1-p^{\prime}} d t\right)^{r / q^{\prime}} v[a, x]^{p^{\prime}} w_{n}(x)^{1-p^{\prime}} d x\right\}^{1 / r} .
\end{aligned}
$$

Next, since by Hölder's inequality,

$$
\begin{aligned}
\int_{a}^{b} \mid & h_{n}(x)-\frac{1}{v[a, b]} \int_{a}^{b} h_{n} d v \mid \mu(x) d x \\
\leq & \left(\int_{a}^{b}\left|h_{n}(x)-\frac{1}{v[a, b]} \int_{a}^{b} h_{n} d v\right|^{q} \mu(x) d x\right)^{1 / q} \mu[a, b]^{1 / q^{\prime}}
\end{aligned}
$$

and

$$
\begin{aligned}
& \int_{a}^{b}\left|\int_{a}^{x} h_{n}^{\prime}(t) v[a, t] d t-\int_{x}^{b} h_{n}^{\prime}(t) v[t, b] d t\right| \mu(x) d x \\
& \quad \geq \frac{1}{2} \int_{\alpha}^{b}\left|\int_{a}^{x} h_{n}^{\prime}(t) v[a, t] d t\right| \mu(x) d x \quad \text { by }(2.12) \\
& \quad=\frac{1}{2} \int_{a}^{b} h_{n}^{\prime}(t) v[a, t]\left(\int_{\max \{t, \alpha\}}^{b} \mu(x) d x\right) d t \quad \text { by Fubini's theorem } \\
& \quad \geq \frac{1}{2} \int_{\alpha}^{b_{n}} \mu[t, b]^{1+r /(p q)} v[a, t]^{p^{\prime}} w_{n}(t)^{1-p^{\prime}} d t \quad \text { by definition of } h_{n}^{\prime},
\end{aligned}
$$

we have by (2.3) for the function $h_{n}$,

$$
\frac{\int_{\alpha}^{b_{n}} \mu[t, b]^{1+r /(p q)} v[a, t]^{p^{\prime}} w_{n}(t)^{1-p^{\prime}} d t}{\left(\int_{\alpha}^{b_{n}} \mu[t, b]^{r / q} v[a, t]^{p^{\prime}} w_{n}(t)^{1-p^{\prime}} d t\right)^{1 / p}} \leq C C_{B} \mu[a, b]^{1 / q^{\prime}} .
$$

Since $1+r /(p q)=r / q-r /\left(p q^{\prime}\right)$, we have

(2.18) $\mu[a, b]^{-r /\left(q q^{\prime}\right)}\left(\int_{\alpha}^{b_{n}} \mu[t, b]^{r / q} v[a, t]^{p^{\prime}} w_{n}(t)^{1-p^{\prime}} d t\right)^{1 / p^{\prime}} \leq C C_{B}$. 
We now let $n \rightarrow \infty$ in (2.17) and obtain

$$
\begin{aligned}
& \left\{\int_{\alpha}^{b_{0}} \mu[x, b]^{r / q}\left(\int_{\alpha}^{x} v[a, t]^{p^{\prime}} w(t)^{1-p^{\prime}} d t\right)^{r / q^{\prime}} v[a, x]^{p^{\prime}} w(x)^{1-p^{\prime}} d x\right\}^{1 / r} \\
& \quad \leq C C_{B}<\infty .
\end{aligned}
$$

Hence,

$$
\begin{aligned}
& \left\{\int_{\alpha}^{b} \mu[x, b]^{r / q}\left(\int_{\alpha}^{x} v[a, t]^{p^{\prime}} w(t)^{1-p^{\prime}} d t\right)^{r / q^{\prime}} v[a, x]^{p^{\prime}} w(x)^{1-p^{\prime}} d x\right\}^{1 / r} \\
& \quad \leq C C_{B}<\infty
\end{aligned}
$$

since the left-hand sides of the previous two estimates are the same. Similarly, from (2.18),

$$
\left\{\int_{\alpha}^{b} \mu[x, b]^{r / q} v[a, x]^{p^{\prime}} w(x)^{1-p^{\prime}} d x\right\}^{1 / r} \leq C C_{B}<\infty .
$$

As we noted before, it then follows that (2.9) holds. Consequently, since we already verified (2.10), (2.7) holds, i.e., the first part of $K_{p, q}(\mu, v, w)$ is finite in case $1<q<p<\infty$. Similarly, we can show that in this case the second part of $K_{p, q}(\mu, v, w)$ is finite, i.e.,

$$
\left(\int_{a}^{b} \mu[a, x]^{r / q}\left(\int_{x}^{b} v[t, b]^{p^{\prime}} w(t)^{1-p^{\prime}} d t\right)^{r / q^{\prime}} v[x, b]^{p^{\prime}} w(x)^{1-p^{\prime}} d x\right)^{1 / r}<\infty .
$$

Thus $K_{p, q}(\mu, v, w)<\infty$ if (1.5) holds and $1<q<p<\infty$.

Finally, a similar argument can be used for the case $q=1,1<p<\infty$, and indeed most of its detail has already been done. This completes the proof of Theorem 1.4.

\section{Remark 2.19.}

1. Note that we can also take $[a, b]$ to be an unbounded interval. The same proof works except that we take $b_{n}$ to be a sequence of numbers increasing to $b_{0}, w_{n}(x)=w(x)+\frac{1}{n}\left(1+|x|^{2 /\left(p^{\prime}-1\right)}\right)$ and we need to assume that the functions $f$ involved are in $L_{v}^{1}[a, b]$ in addition to being Lipschitz continuous.

2. In case $p=1$ and $q=\infty$, we note that

$$
\left|f(x)-\int_{a}^{b} f d v / v[a, b]\right| \leq K_{1, \infty}(1, v, w) \int_{a}^{b}\left|f^{\prime}(y)\right| w(y) d y
$$

for all $x$ in $[a, b]$ 
for all Lipschitz continuous functions (and hence all absolutely continuous functions) $f$ on $[a, b]$, where

$$
K_{1, \infty}(1, v, w)=\left\|\max \{v[a, .], v[., b]\} w^{-1}\right\|_{L^{\infty}[a, b]} .
$$

Moreover, $K_{1, \infty}(1, v, w)$ is the smallest possible constant for the inequality.

Proof. The inequality easily follows from (1.9). For the converse, a simple modification of the case $p=1,1 \leq q<\infty$ in the proof of Theorem 1.4 (using the same $f_{\varepsilon}$ ) will do the job.

3. Extension to 2-Dimensional Poincaré inequalities. We can now generalize the 1-dimensional inequalities to 2-dimensional ones by standard methods. Of course the method can also give results for $n$ dimensions, but to simplify the notation, we will only consider $n=2$. First, let us define some notation. Let $1 \leq q_{1}, q_{2}<\infty$. If $E$ is a measurable set in $\mathbb{R} \times \mathbb{R}$, and $w$ is a weight of the form $w(x, y)=w_{1}(x) w_{2}(y)$, we write $w=w_{1} \times w_{2}$ and say that $w$ is a product weight. We then define

$$
\|f\|_{L_{w_{1} \times w_{2}}^{\left(q_{1}, q_{2}\right)}(E)}=\left(\int_{\mathbb{R}}\left[\int_{\mathbb{R}}|f(x, y)|^{q_{1}} \chi_{E}(x, y) w_{1}(x) d x\right]^{q_{2} / q_{1}} w_{2}(y) d y\right)^{1 / q_{2}}
$$

and

$\|f\|_{L_{w_{1} \times w_{2}}^{\left(\widehat{q_{1}, q_{2}}\right)}(E)}=\left(\int_{\mathbb{R}}\left[\int_{\mathbb{R}}|f(x, y)|^{q_{2}} \chi_{E}(x, y) w_{2}(y) d y\right]^{q_{1} / q_{2}} w_{1}(x) d x\right)^{1 / q_{1}}$.

If $v=v_{1} \times v_{2}$ is a product weight and $I \times J$ is a fixed rectangle in $\mathbb{R}^{2}$, we use the notation

$$
f_{v, a v}=\frac{1}{v(I \times J)} \int_{I \times J} f\left(y_{1}, y_{2}\right) v_{1}\left(y_{1}\right) v_{2}\left(y_{2}\right) d y_{1} d y_{2}
$$

for the integral average of $f$ over $I \times J$ with respect to $v$.

We now state a special type of Poincaré inequality in 2-dimensions, using the same notation as in Theorem 1.4. 
Theorem 3.1 Let $I \times J=[a, b] \times[c, d]$. Let $\mu=\mu_{1} \times \mu_{2}, v=v_{1} \times v_{2}$, $w=w_{1} \times w_{2}$ be product weights on $I \times J$ such that $\mu, v \in L^{1}(I \times J)$. Let $1 \leq p_{1}, p_{2}, q_{1}, q_{2}, s_{1}<\infty$. Then

$$
\begin{aligned}
& \left\|f-f_{v, a v}\right\|_{L_{\mu_{1} \times \mu_{2}}^{\left(a_{1}, q_{2}\right)}(I \times J)} \\
& \quad \leq C_{1}\left\|\frac{\partial f}{\partial x_{1}}\right\|_{L_{w_{1} \times \mu_{2}}^{\left(p_{1}, q_{2}\right)}(I \times J)}+C_{2} \frac{\mu_{1}(I)^{1 / q_{1}}}{v_{1}(I)^{1 / s_{1}}}\left\|\frac{\partial f}{\partial x_{2}}\right\|_{L_{v_{1} \times w_{2}}^{\left(\widehat{\left(\widehat{1}, p_{2}\right)}(I \times J)\right.}}
\end{aligned}
$$

for all Lipschitz continuous functions $f$ on $I \times J$ if and only if both

$$
K_{p_{1}, q_{1}}\left(\mu_{1}, v_{1}, w_{1}\right)<\infty \quad \text { and } \quad K_{p_{2}, q_{2}}\left(\mu_{2}, v_{2}, w_{2}\right)<\infty .
$$

Moreover, if $C_{1}$ and $C_{2}$ are the best constants for (3.2) for all $f$, then

$$
\begin{array}{rlrl}
\frac{K_{p_{i}, q_{i}}\left(\mu_{i}, v_{i}, w_{i}\right)}{2} \leq C_{i} \leq C_{0}\left(p_{i}, q_{i}\right) K_{p_{i}, q_{i}}\left(\mu_{i}, v_{i}, w_{i}\right) & & \text { if } q_{i} \geq p_{i}>1, \\
C_{i} \leq C_{0}\left(p_{i}, q_{i}\right) K_{p_{i}, q_{i}}\left(\mu_{i}, v_{i}, w_{i}\right) & & \text { if } p_{i}>q_{i} \geq 1, \\
C_{i} & =K_{1, q_{i}}\left(\mu_{i}, v_{i}, w_{i}\right) & & \text { if } p_{i}=1,
\end{array}
$$

where $C_{0}\left(p_{i}, q_{i}\right)$ is as usual.

Proof. We will take $C_{0}\left(1, q_{1}\right)=C_{0}\left(1, q_{2}\right)=1$. By the triangle inequality,

$$
\begin{aligned}
& \left(\int _ { J } \left[\int_{I}\left|f\left(x_{1}, x_{2}\right)-\frac{1}{v_{1}(I) v_{2}(J)} \int_{I \times J} f\left(y_{1}, y_{2}\right) v_{1}\left(y_{1}\right) v_{2}\left(y_{2}\right) d y_{1} d y_{2}\right|^{q_{1}}\right.\right. \\
& \left.\left.\cdot \mu_{1}\left(x_{1}\right) d x_{1}\right]^{q_{2} / q_{1}} \mu_{2}\left(x_{2}\right) d x_{2}\right)^{1 / q_{2}} \\
& \leq\left(\int_{J}\left[\int_{I}\left|f\left(x_{1}, x_{2}\right)-\frac{1}{v_{1}(I)} \int_{I} f\left(y_{1}, x_{2}\right) v_{1}\left(y_{1}\right) d y_{1}\right|^{q_{1}} \mu_{1}\left(x_{1}\right) d x_{1}\right]^{q_{2} / q_{1}}\right. \\
& +\left(\int_{J}\left[\int_{I}\left|\frac{1}{v_{1}(I)} \int_{I} f\left(y_{1}, x_{2}\right) v_{1}\left(y_{1}\right) d y_{1}-f_{v, a v}\right|^{q_{1}} \mu_{1}\left(x_{1}\right) d x_{1}\right]^{q_{2} / q_{1}}\right)^{1 / q_{2}} \\
& =S+T .
\end{aligned}
$$

Next observe that by Theorem 1.4 , 


$$
\begin{gathered}
S \leq\left(\int_{J} C_{0}\left(p_{1}, q_{1}\right)^{q_{2}} K_{p_{1}, q_{1}}\left(\mu_{1}, v_{1}, w_{1}\right)^{q_{2}}\right. \\
\left.\cdot\left[\int_{I}\left|\frac{\partial f}{\partial x_{1}}\right|^{p_{1}} w_{1}\left(x_{1}\right) d x_{1}\right]^{q_{2} / p_{1}} \mu_{2}\left(x_{2}\right) d x_{2}\right)^{1 / q_{2}} \\
=C_{1}\left(\int_{J}\left[\int_{I}\left|\frac{\partial f}{\partial x_{1}}\right|^{p_{1}} w_{1}\left(x_{1}\right) d x_{1}\right]^{q_{2} / p_{1}} \mu_{2}\left(x_{2}\right) d x_{2}\right)^{1 / q_{2}} .
\end{gathered}
$$

Also, by Minkowski's and Hölder's inequalities,

$$
\begin{aligned}
& T \leq \frac{1}{v_{1}(I)}\left(\int _ { J } \left[\int _ { I } \left(\int_{I} \mid f\left(y_{1}, x_{2}\right)\right.\right.\right. \\
& \left.-\frac{1}{v_{2}(J)} \int_{J} f\left(y_{1}, y_{2}\right) v_{2}\left(y_{2}\right) d y_{2} \mid v_{1}\left(y_{1}\right) d y_{1}\right)^{q_{1}} \\
& \left.\left.\mu_{1}\left(x_{1}\right) d x_{1}\right]^{q_{2} / q_{1}} \mu_{2}\left(x_{2}\right) d x_{2}\right)^{1 / q_{2}} \\
& =\frac{\mu_{1}(I)^{1 / q_{1}}}{v_{1}(I)}\left(\int _ { J } \left[\int_{I} \mid f\left(y_{1}, x_{2}\right)\right.\right. \\
& \left.-\frac{1}{v_{2}(J)} \int_{J} f\left(y_{1}, y_{2}\right) v_{2}\left(y_{2}\right) d y_{2} \mid v_{1}\left(y_{1}\right) d y_{1}\right]^{q_{2}} \\
& \left.\mu_{2}\left(x_{2}\right) d x_{2}\right)^{1 / q_{2}} \\
& \leq \frac{\mu_{1}(I)^{1 / q_{1}}}{v_{1}(I)} \int_{I}\left[\int_{J} \mid f\left(y_{1}, x_{2}\right)\right. \\
& \left.-\left.\frac{1}{v_{2}(J)} \int_{J} f\left(y_{1}, y_{2}\right) v_{2}\left(y_{2}\right) d y_{2}\right|^{q_{2}} \mu_{2}\left(x_{2}\right) d x_{2}\right]^{1 / q_{2}} v_{1}\left(y_{1}\right) d y_{1} \\
& \leq \frac{\mu_{1}(I)^{1 / q_{1}}}{v_{1}(I)}\left[\int_{I} C_{0}\left(p_{2}, q_{2}\right) K_{p_{2}, q_{2}}\left(\mu_{2}, v_{2}, w_{2}\right)\left(\int_{J}\left|\frac{\partial f}{\partial x_{2}}\right|^{p_{2}} w_{2}\left(x_{2}\right) d x_{2}\right)^{1 / p_{2}}\right. \\
& =C_{2} \frac{\mu_{1}(I)^{1 / q_{1}}}{v_{1}(I)}\left\|\frac{\partial f}{\partial x_{2}}\right\|_{L_{v_{1} \times w_{2}}^{\left(1, p_{2}\right)}(I \times J)} \leq C_{2} \frac{\mu_{1}(I)^{1 / q_{1}}}{v_{1}(I)^{1 / s_{1}}}\left\|\frac{\partial f}{\partial x_{2}}\right\|_{L_{v_{1} \times w_{2}}^{\left(\widehat{s}_{1}, p_{2}\right)}(I \times J)} .
\end{aligned}
$$

The converse follows from the one variable case by considering separately functions $f\left(x_{1}, x_{2}\right)$ depending only on $x_{1}$ or only on $x_{2}$. This completes the proof of our theorem. 
The previous theorem has some interesting special cases, such as when $q_{1}=q_{2}=p_{1}, s_{1}=p_{2}$ and when $p_{1}=p_{2}=q_{1}=q_{2}=s_{1}, \mu_{1} \times \mu_{2}=v_{1} \times v_{2}=$ $w_{1} \times w_{2}$. We now list these results. We will use the notation

$$
L_{\mu_{1} \times \mu_{2}}^{p_{1}}(I \times J)=L_{\mu_{1} \times \mu_{2}}^{\left(p_{1}, p_{1}\right)}(I \times J),
$$

and

$$
L_{v_{1} \times w_{2}}^{p_{2}}(I \times J)=L_{v_{1} \times w_{2}}^{\left(p_{2}, p_{2}\right)}(I \times J)=L_{v_{1} \times w_{2}}^{\left(\widehat{p_{2}, p_{2}}\right)}(I \times J) .
$$

Theorem 3.3 Let $I \times J$ be a rectangle in $\mathbb{R}^{2}$. Let $\mu=\mu_{1} \times \mu_{2}, v=v_{1} \times v_{2}$, $w=w_{1} \times w_{2}$ be product weights on $I \times J$ such that $\mu, v \in L^{1}(I \times J)$. Let $1 \leq p_{1}$, $p_{2}<\infty$. Then

$$
\begin{aligned}
& \left\|f-f_{v, a v}\right\|_{L_{\mu_{1} \times \mu_{2}}^{p_{1}(I \times J)}} \\
& \quad \leq C_{1}\left\|\frac{\partial f}{\partial x_{1}}\right\|_{L_{w_{1} \times \mu_{2}}^{p_{1}(I \times J)}}+C_{2} \frac{\mu_{1}(I)^{1 / p_{1}}}{v_{1}(I)^{1 / p_{2}}}\left\|\frac{\partial f}{\partial x_{2}}\right\|_{L_{v_{1} \times w_{2}}^{p_{2}(I \times J)}}
\end{aligned}
$$

for all Lipschitz continuous functions $f$ on $I \times J$ if and only if both $K_{p_{1}, p_{1}}\left(\mu_{1}, v_{1}, w_{1}\right)<\infty$ and $K_{p_{2}, p_{1}}\left(\mu_{2}, v_{2}, w_{2}\right)<\infty$. Moreover, if $C_{1}$ and $C_{2}$ are the best constants for the inequality for all $f$, then

$$
\begin{aligned}
\frac{K_{p_{i}, p_{1}}\left(\mu_{i}, v_{i}, w_{i}\right)}{2} & \leq C_{i} \leq C_{0}\left(p_{i}, p_{1}\right) K_{p_{i}, p_{1}}\left(\mu_{i}, v_{i}, w_{i}\right) & & \text { if } p_{1} \geq p_{i}>1, \\
C_{i} & \leq C_{0}\left(p_{i}, p_{1}\right) K_{p_{i}, p_{1}}\left(\mu_{i}, v_{i}, w_{i}\right) & & \text { if } p_{i}>p_{1} \geq 1, \\
C_{i} & =K_{1, p_{1}}\left(\mu_{i}, v_{i}, w_{i}\right) & & \text { if } p_{i}=1,
\end{aligned}
$$

where $C_{0}\left(p_{i}, p_{1}\right)$ is as usual.

For the second case, since it can be easily stated in the case of $\mathbb{R}^{n}$, we will do so.

Theorem 3.5 Let $R$ be a parallelepiped in $\mathbb{R}^{n}$ and $1 \leq p<\infty$. If $w=$ $w_{1} \times w_{2} \times \cdots \times w_{n}$ is a product weight in $L^{1}(R)$, then

$$
\left\|f-f_{w, a v}\right\|_{L_{w}^{p}(R)} \leq \sum_{i=1}^{n} C_{i}\left\|\frac{\partial f}{\partial x_{i}}\right\|_{L_{w}^{p}(R)}
$$

for all Lipschitz continuous functions $f$ on $R$ if and only if all $K_{p, p}\left(w_{i}, w_{i}, w_{i}\right)<$ $\infty$ for all $i$. Moreover, if $C_{i}$ 's are the best constants for the inequality for all $f$, then

$$
\begin{aligned}
\frac{K_{p, p}\left(w_{i}, w_{i}, w_{i}\right)}{2} & \leq C_{i} \leq p^{1 / p}\left(p^{\prime}\right)^{1 / p^{\prime}} K_{p, p}\left(w_{i}, w_{i}, w_{i}\right) & & \text { if } p>1, \\
C_{i} & =K_{1,1}\left(w_{i}, w_{i}, w_{i}\right) & & \text { if } p=1 .
\end{aligned}
$$


4. ApPENDIX. We now compute some values of $K_{1, q}(1,1,1)$, that is when $\mu, v$, and $w$ are equal to 1 (the case of Lebesgue measure).

\section{Lemma 4.1}

$$
\max _{0 \leq t \leq 1} t^{q}(1-t)+(1-t)^{q} t \begin{cases}=\frac{1}{2^{q}} & \text { if } 1 \leq q \leq 3 \\ >\frac{1}{2^{q}} & \text { if } q>3\end{cases}
$$

Proof. First note that it is obvious by setting $t=\frac{1}{2}$ that the maximum is at least $\left(\frac{1}{2}\right)^{q}$. We now show the converse. First, let us consider the case $1 \leq q \leq 2$. Note that

$$
t^{q}(1-t)+t(1-t)^{q}=t(1-t)\left(t^{q-1}+(1-t)^{q-1}\right) .
$$

As can be seen easily by differentiation,

$$
t(1-t) \leq \frac{1}{4} \quad \text { and } \quad t^{q-1}+(1-t)^{q-1} \leq 2\left(\frac{1}{2}\right)^{q-1}
$$

since $0 \leq q-1 \leq 1$, and the result follows.

Next let us look at the case $2<q \leq 3$. Clearly, it suffices to show that if $0<t<1$, then $(2 t)^{q}(2-2 t)+(2-2 t)^{q}(2 t) \leq 2$. Hence, letting $x=|1-2 t|$, it suffices to show that $(1+x)(1-x)^{q}+(1+x)^{q}(1-x) \leq 2$ for $0<x<1$. Let $f(x)=(1+x)^{q}$ and $g(x)=(1-x)^{q}$.

$$
\begin{aligned}
f(x)=1+q x+\frac{q(q-1)}{2} x^{2}+\frac{q(q-1)(q-2)}{6} x^{3}+\frac{f^{(4)}(\xi)}{24} x^{4} & \text { for some } \xi \in(0, x), \\
g(x)=1-q x+\frac{q(q-1)}{2} x^{2}-\frac{q(q-1)(q-2)}{6} x^{3}+\frac{g^{(4)}(\tau)}{24} x^{4} & \text { for some } \tau \in(0, x) .
\end{aligned}
$$

Moreover,

$$
f^{(4)}(x), g^{(4)}(x) \leq 0 \quad \text { for } 0<x<1,
$$

since $q \leq 3$. Hence,

$$
\begin{aligned}
& (1+x)(1-x)^{q}+(1+x)^{q}(1-x) \\
& \quad \leq(1+x)\left(1-q x+\frac{q(q-1)}{2} x^{2}-\frac{q(q-1)(q-2)}{6} x^{3}\right)+
\end{aligned}
$$




$$
\begin{aligned}
& +(1-x)\left(1+q x+\frac{q(q-1)}{2} x^{2}+\frac{q(q-1)(q-2)}{6} x^{3}\right) \\
= & 2+q(q-3) x^{2}-\frac{q(q-1)(q-2)}{3} x^{4} \leq 2
\end{aligned}
$$

since $q \leq 3$. Finally, note that $f^{(4)}(x), g^{(4)}(x) \geq 0$ for $0<x<1$ when $q>3$. Hence,

$$
(1+x)(1-x)^{q}+(1+x)^{q}(1-x) \geq 2+q(q-3) x^{2}-\frac{q(q-1)(q-2)}{3} x^{4}
$$

which is strictly greater than 2 for some $0<x<1$. This completes the proof of the lemma.

It is now easy to see that $K_{1, q}(1,1,1)=\frac{1}{2}(b-a)^{1 / q}$ for $1 \leq q \leq 3$ and $K_{1, q}(1,1,1)>\frac{1}{2}(b-a)^{1 / q}$ for $q>3$.

Finally, let us discuss an application of our main theorem to a problem of differential equations. While Hardy-type inequalities can be applied to initial value problems for ordinary differential equations, our theorem can be applied to ordinary differential equations with given average value.

Theorem 4.2 Let $1<p<\infty, M, K>0,-\infty<a<b<\infty$, and $|b-a| \leq K / M$. Suppose

$$
\text { (A) }\left\{\begin{array}{r}
|F(x, y)| \leq M \\
\text { for } x \in[a, b] \text { and }\left|y-y_{0}\right| \leq K, \\
\left|F\left(x, y_{1}\right)-F\left(x, y_{2}\right)\right| \leq|g(x)|\left|y_{1}-y_{2}\right| \\
\quad \text { for } x \in[a, b], y_{1}, y_{2} \in\left[y_{0}-K, y_{0}+K\right] .
\end{array}\right.
$$

Let $\sigma$ be a nonnegative weight and $v$ be a nonnegative measure on $[a, b]$ such that $v[a, b]>0$. If

$$
\lambda=K_{1, p^{\prime}}(\mu, v, 1)\|g\|_{L_{\sigma}^{p}[a, b]}<1, \quad \mu=\sigma^{1-p^{\prime}},
$$

then the ordinary differential equation $u^{\prime}(x)=F(x, u(x)), x \in[a, b]$, has a unique absolutely continuous solution $u:[a, b] \rightarrow\left[y_{0}-K, y_{0}+K\right]$ such that $\int_{a}^{b} u d v / v[a, b]=y_{0}$.

Proof. Let $u_{1}(x)=y_{0}$ on $[a, b]$ and for each $n \in \mathbf{N}$, define

$u_{n+1}(x)=y_{0}+\frac{1}{v[a, b]}\left[\int_{a}^{x} v[a, t] F\left(t, u_{n}(t)\right) d t-\int_{x}^{b} v[t, b] F\left(t, u_{n}(t)\right) d t\right]$ for $x \in[a, b]$. 
Note that $\left|u_{1}(x)-y_{0}\right|=0 \leq K$ and

$$
\left|u_{n+1}(x)-y_{0}\right| \leq \int_{a}^{b}\left|F\left(t, u_{n}(t)\right)\right| d t \leq M(b-a) \leq K,
$$

by induction. Also, observe that $u_{n+1}^{\prime}(x)=F\left(x, u_{n}(x)\right)$ almost everywhere, and hence by (1.9) we get $1 /(v[a, b]) \int_{a}^{b} u_{n+1} d v=y_{0}$. So by Theorem 1.4 and then Hölder's inequality, we have

$$
\begin{aligned}
\left\|u_{n+1}-u_{n}\right\|_{L_{\mu}^{p^{\prime}}[a, b]} & \leq K_{1, p^{\prime}}(\mu, v, 1)\left\|u_{n+1}^{\prime}-u_{n}^{\prime}\right\|_{L^{1}[a, b]} \\
& =K_{1, p^{\prime}}(\mu, v, 1)\left\|F\left(., u_{n}(.)\right)-F\left(., u_{n-1}(.)\right)\right\|_{L^{1}[a, b]} \\
& \leq K_{1, p^{\prime}}(\mu, v, 1)\|g\|_{L_{\sigma}^{p}[a, b]}\left\|u_{n}-u_{n-1}\right\|_{L_{\mu}^{p^{\prime}}[a, b]} \\
& =\lambda\left\|u_{n}-u_{n-1}\right\|_{L_{\mu}^{p^{\prime}}[a, b]} .
\end{aligned}
$$

Since

$$
\left\|u_{2}-u_{1}\right\|_{L_{\mu}^{p^{\prime}[a, b]}}=\left\|u_{2}-y_{0}\right\|_{L_{\mu}^{p^{\prime}[a, b]}} \leq K_{1, p^{\prime}}(\mu, v, 1)\left\|F\left(., y_{0}\right)\right\|_{L^{1}[a, b]}<\infty
$$

by Theorem 1.4 , clearly $\left\{u_{n}\right\}$ is a Cauchy sequence in $L_{\mu}^{p^{\prime}}[a, b]$. Thus there exists $u \in L_{\mu}^{p^{\prime}}[a, b]$ such that $u_{n} \rightarrow u$ in $L_{\mu}^{p^{\prime}}[a, b]$ and hence there is a subsequence $\left\{u_{n_{k}}\right\} \rightarrow u$ almost everywhere. Moreover note that by Hölder's inequality,

$$
\int_{a}^{b}\left|F\left(t, u_{n_{k}-1}(t)\right)-F(t, u(t))\right| d t \leq\|g\|_{L_{\sigma}^{p}[a, b]}\left\|u_{n_{k}-1}-u\right\|_{L_{\mu}^{p^{\prime}}[a, b]} \rightarrow 0 .
$$

Hence,

$$
\begin{aligned}
u(x) & =\lim _{k \rightarrow \infty} u_{n_{k}}(x) \\
& =y_{0}+\frac{1}{v[a, b]}\left[\int_{a}^{x} v[a, t] F(t, u(t)) d t-\int_{x}^{b} v[t, b] F(t, u(t)) d t\right]
\end{aligned}
$$

for almost every $x \in[a, b]$. So if we redefine $u(x)$ to be the right-hand side of the above equation then $u$ is absolutely continuous, $\left|u(x)-y_{0}\right| \leq K$ and $u^{\prime}(x)=F(x, u(x))$ almost everywhere.

Finally, let us prove the uniqueness of the solution. Suppose $u$ and $u_{0}$ are absolutely continuous functions and $u, u_{0}:[a, b] \rightarrow\left[y_{0}-K, y_{0}+K\right]$ such that $u^{\prime}(x)=F(x, u(x)), u_{0}^{\prime}(x)=F\left(x, u_{0}(x)\right)$ for almost all $x$ in $[a, b]$ and $\int_{a}^{b} u d v=\int_{a}^{b} u_{0} d v=y_{0} v[a, b]$. Then

$$
\begin{aligned}
\left\|u-u_{0}\right\|_{L_{\mu}^{p^{\prime}}[a, b]} & \leq K_{1, p^{\prime}}(\mu, v, 1)\left\|F(., u(.))-F\left(., u_{0}(.)\right)\right\|_{L^{1}[a, b]} \\
& \leq \lambda\left\|u-u_{0}\right\|_{L_{\mu}^{p^{\prime}}[a, b]} .
\end{aligned}
$$


But

$$
\left\|u-u_{0}\right\|_{L_{\mu}^{p^{\prime}}[a, b]} \leq\left\|u-y_{0}\right\|_{L_{\mu}^{p^{\prime}}[a, b]}+\left\|u_{0}-y_{0}\right\|_{L_{\mu}^{p^{\prime}}[a, b]}
$$

is finite by Theorem 1.4, as $F$ is bounded. Also $\lambda<1$ and hence $u=u_{0}$ almost everywhere.

\section{Remarks.}

1. In case $v$ is a Delta measure at $a$, our problem is just the usual initial value problem.

2. In case $p=1$ or $\infty$, it is easy to check that our theorem still holds for $\sigma=1$.

3. Using almost the same idea as in the proof of the previous theorem, we can also obtain the following result:

Theorem 4.3 Under the same assumptions as in Theorem 4.2, but with conditions (A) replaced by

$\left(A^{\prime}\right)\left\{\begin{array}{l}\left|F\left(x, y_{0}\right)\right| \in L^{1}[a, b], \\ \left|F\left(x, y_{1}\right)-F\left(x, y_{2}\right)\right| \leq|g(x)|\left|y_{1}-y_{2}\right| \quad \text { for } x \in[a, b], y_{1}, y_{2} \in \mathbb{R},\end{array}\right.$ there is an absolutely continuous function $u$ on $[a, b]$ such that $\int_{a}^{b} u d v / v[a, b]=$ $y_{0}$ and $u^{\prime}(x)=F(x, u(x))$ almost everywhere. Moreover, the solution is unique if $\mu=\sigma^{1-p^{\prime}} \in L^{1}[a, b]$.

\section{REFERENCES}

[1] F. J. Almgren \& E. H. Lieb, Symmetric rearrangement is sometimes continuous, J. Amer. Math. Soc. 2 (1989), 683-773.

[2] C. J. AMick, Some remarks on Rellich's theorem and the Poincare inequality, J. London Math. Soc. 18 (1978), 81-93.

[3] K. F. ANDERSON \& E. T. SAWYER, Weighted norm inequalities for the Riemann-Liouville and Weyl fractional integral operators, Trans. Amer. Math. Soc. 308 (1988), 547-558.

[4] T. Aubin, Problèmes isopérimétriques et espaces de Sobolev, J. Diff. Geometry 11 (1976), 573-598.

[5] B. BOJARSKI, Remarks on Sobolev imbedding inequalities, Complex Analysis, Lecture Notes in Math., 1351, Springer-Verlag, 1989, 52-68.

[6] H. J. BRASCAMP \& E. H. LIEB, Best constants in Young's inequality, its converse, and its generalization to more than three functions, Adv. in Math. 20 (1976), 151-173.

[7] H. J. Brascamp, E. H. Lieb \& J. M. LutTinger, A general rearrangement inequality for multiple integrals, J. Funct. Anal. 17 (1974), 227-237. 
[8] E. CARLEN \& M. LOSS, Extremals of functionals with competing symmetries, J. Funct. Anal. 88 (1990), 437-456.

[9] C. CARTON-LEBRUN \& H. P. HeINIG, Weighted norm inequalities involving gradients, Proc. Roy. Soc. Edinburgh Sect. A 112 (1989), 331-341.

[10] S. Chanillo \& R. L. Wheeden, Poincaré inequalities for a class of non-Ap weights, Indiana Math. J. 41 (1992), 605-623.

[11] F. Chiarenza \& M. Frasca, A note on a weighted Sobolev inequality, Proc. Amer. Math. Soc., 93 (1985), 703-704.

[12] S. K. ChuA, Extension theorems on weighted Sobolev spaces, Indiana Math. J., 41 (1992), 1027-1076.

[13] S. K. CHUA, Weighted Sobolev's inequalities on domains satisfying the chain condition, Proc. Amer. Math. Soc., 117 (1993), 449-457.

[14] S. K. CHUA, Weighted Sobolev's inequalities on certain domains, J. London Math. Soc. (2) 51 (1995) 532-544.

[15] S. K. CHUA, Weighted Sobolev inequalities of mixed norm, Real Anal. Exchange, 21 (1995/96), 555-571.

[16] A. Cianchi, D. E. Edmunds \& P. Gurka, On weighted Poincaré inequalities, Math. Nachr., 180 (1996), 15-41.

[17] D. E. Edmunds \& W. D. Evans, Spectral Theory and Differential Operators, Oxford University Press, 1987.

[18] D. E. Edmunds \& B. OpIC, Weighted Poincaré and Friedrichs inequalities, J. London Math. Soc. 47 (1993), 79-96.

[19] D. E. Edmunds, B. OPIC \& L. PICK, Poincaré and Friedrichs inequalities in abstract Sobolev spaces, Math. Proc. Comb. Phil. Soc. 113 (1993), 355-379.

[20] D. E. Edmunds, B. OpIC \& J. RÁKOSNÍK, Poincaré and Friedrichs inequalities in abstract Sobolev spaces II, Math. Proc. Camb. Phil. Soc. 115 (1994), 159-173.

[21] E. B. Fabes, C. E. KenIG \& R. P. SERAPIONI, The local regularity of solutions of degenerate elliptic equations, Comm. P.D.E. 7 (1982), 77-116.

[22] B. Franchi, C. E. Gutiérrez \& R. L. WheEden, Weighted Sobolev-Poincaré inequalities for Grushin type operators, Comm. P.D.E. 19 (1994), 523-604.

[23] P. GURKa \& A. KUfNer, A note on a two-weighted Sobolev inequality, Approximation and Function Spaces, 169-172, Banach center publ. 22, Warsaw 1989.

[24] A. Kufner, Weighted Sobolev Spaces, John Wiley \& Sons Ltd., 1985.

[25] A. Kufner \& B. OPIC, Hardy-type Inequalities, Pitman Research Notes 219, Longman House, U.K., 1990.

[26] E. H. LIEB, Gaussian kernels have only Gaussian maximizers, Invent. Math. 102 (1990), 179-208.

[27] E. H. LiEb, Sharp constants in the Hardy-Littlewood-Sobolev and related inequalities, Ann. of Math. 18 (1983), 349-374.

[28] G. LU \& R. L. WHEEDEN, Poincaré inequalities, isoperimetric estimates and representation formulas on product spaces, Indiana Math. J., 47 (1998), 123-151.

[29] V. G. MAZ'JA, Sobolev Spaces, Springer-Verlag, New York, 1985.

[30] E. T. SAWYER \& R. L. WHEEDEN, Weighted inequalities for fractional integrals on Euclidean and homogeneous spaces, Amer. J. Math., 114 (1992), 813-874.

[31] G. Sinnamon, A weighted gradient inequality, Proc. Roy. Soc., Edinburgh Sect. A 111 (1989), 329-335.

[32] G. Sinnamon, Weighted Hardy and Opial type inequalities, J. Math. Anal. Appl., 160 (1991), 434-445. 
[33] G. Sinnamon, Best constant in Sobolev inequality, Ann. Mat. Pura Appl. 110 (1976), 353-372.

[34] G. TAlenti, Some inequalities of Sobolev type on two-dimensional spheres, General inequalities 5, 5th International Conference on General Inequalities, Oberwolfach (1986), 401-408.

[35] A. Torchinsky, Real-Variable Methods in Harmonic Analysis, Academic Press Inc., New York, 1986.

[36] W. ZIEMER, Weakly Differentiable Functions, Springer-Verlag, 1989.

SENG-KeE CHUA

Department of Mathematics

National University of Singapore

10, Kent Ridge Crescent

Singapore 119260

EMAIL: matcsk@math.nus.edu.sg

RICHARD L. WHEEDEN

Department of Mathematics

Rutgers University

New Brunswick, NJ 08903

EMAIL: wheeden@math.rutgers.edu

Subject Classification: Primary 26D10, 46E35

KEYWORDs: Poincaré inequalities, Sobolev's inequalities, Hardy inequalities, $A_{p}$ weights

Submitted: February 2nd, 1999; revised: July 14th, 1999 